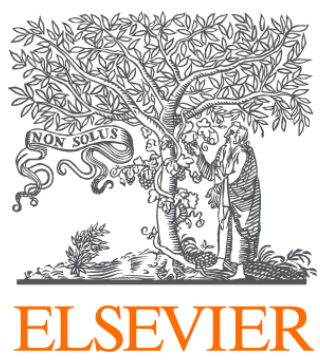

Since January 2020 Elsevier has created a COVID-19 resource centre with free information in English and Mandarin on the novel coronavirus COVID-

19. The COVID-19 resource centre is hosted on Elsevier Connect, the company's public news and information website.

Elsevier hereby grants permission to make all its COVID-19-related research that is available on the COVID-19 resource centre - including this research content - immediately available in PubMed Central and other publicly funded repositories, such as the WHO COVID database with rights for unrestricted research re-use and analyses in any form or by any means with acknowledgement of the original source. These permissions are granted for free by Elsevier for as long as the COVID-19 resource centre remains active. 


\section{International Society for Heart and Lung Transplantation consensus statement for the standardization of bronchoalveolar lavage in lung transplantation}

Tereza Martinu, MD, ${ }^{a, 1}$ Angela Koutsokera, MD, PhD, , , c,1 Christian Benden, MD, FCCP, ${ }^{d}$ Edward Cantu, MD, ${ }^{e}$ Daniel Chambers, MD, ${ }^{f}$

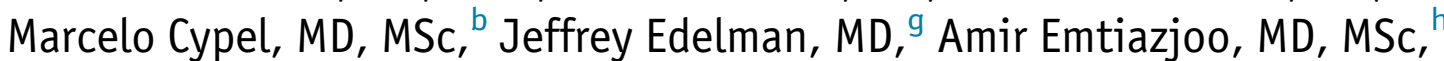
Andrew J. Fisher, PhD, FRCP, ${ }^{i}$ John R. Greenland, MD, PhD, ${ }^{j}$ Don Hayes, Jr, MD, MS, MEd, ${ }^{k}$ David Hwang, MD, PhD, FRCP, Brian C. Keller, MD, PhD, ${ }^{\mathrm{m}}$ Erika D. Lease, MD, ${ }^{\mathrm{n}}$ Michael Perch, MD, ${ }^{\circ}$ Masaaki Sato, MD, PhD, ${ }^{p}$ Jamie L. Todd, MD, ${ }^{q}$ Stijn Verleden, PhD, ${ }^{r}$ Jan von der Thüsen, MA, MBBS, PhD, ${ }^{\mathrm{s}} \mathrm{S}$. Samuel Weigt, MD, MS, ${ }^{\mathrm{t}}$ and Shaf Keshavjee, MD, MSc, FRCSC, FACS ${ }^{b}$, on behalf of the bronchoalveolar lavage standardization workgroup

From the ${ }^{a}$ Toronto Lung Transplant Program, University Health Network, University of Toronto, Toronto, Ontario, Canada; ${ }^{b}$ Lung Transplant Program, Toronto General Hospital, University Health Network, Toronto, Ontario, Canada; ${ }^{c}$ Lung Transplant Program, Division of Pulmonology, Lausanne University Hospital, Lausanne, Switzerland; ${ }^{d}$ University of Zurich Faculty of Medicine, Zurich, Switzerland; ${ }^{e}$ Department of Surgery, Hospital of the University of Pennsylvania, Philadelphia, Pennsylvania; ${ }^{f}$ Lung Transplant Program, The Prince Charles Hospital, Brisbane, Queensland, Australia; ${ }^{g}$ Lung Transplant Program, Puget Sound VA Medical Center, Seattle, Washington; ${ }^{h}$ Lung Transplant Program, University of Florida, Gainesville, Florida; ${ }^{i}$ Institute of Transplantation, Newcastle Upon Tyne Hospitals and Newcastle University, United Kingdom; ${ }^{j}$ Department of Medicine, VA Health Care System, San Francisco, California; ${ }^{k}$ Lung Transplant Program, Cincinnati Children's Hospital Medical Center, Cincinnati, Ohio; 'Department of Pathology, Toronto General Hospital, University Health Network, Toronto, Ontario, Canada; ${ }^{m}$ Lung Transplant Program, Wexner Medical Center, The Ohio State University, Columbus, Ohio; ${ }^{n}$ Division of Pulmonary, Critical Care, and Sleep Medicine, University of Washington, Seattle, Washington; ${ }^{o}$ Lung Transplant Program, Rigshospitalet, Copenhagen, Denmark; ${ }^{p}$ Department of Surgery, University of Tokyo, Tokyo, Japan; ${ }^{q}$ Lung Transplant Program, Duke University Medical Center, Durham, North Caro-

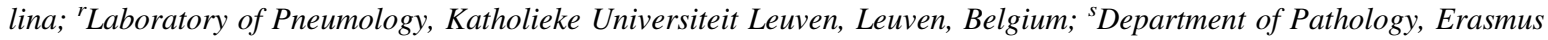
Medical Center, Rotterdam, Netherlands; and the ${ }^{t}$ Lung Transplant Program, University of California Los Angeles, Los Angeles, California.

\section{KEYWORDS:}

lung transplantation; bronchoalveolar lavage; standardization; methodology;
Bronchoalveolar lavage (BAL) is a key clinical and research tool in lung transplantation (LTx). However, BAL collection and processing are not standardized across LTx centers. This International Society for Heart and Lung Transplantation-supported consensus document on BAL standardization aims to clarify definitions and propose common approaches to improve clinical and research practice standards. The following 9 areas are covered: (1) bronchoscopy procedure and BAL collection, (2) sample handling, (3) sample processing for microbiology, (4) cytology, (5) research, (6) microbiome, (7)

\footnotetext{
${ }^{1}$ These authors contributed equally to this work.

Reprint requests: Tereza Martinu, MD, Division of Respirology and Lung Transplant Program, Toronto General Hospital and University Health
}

Network, 585 University Avenue, PMB 11-128, Toronto, ON, Canada, M5G 2N2. Telephone: 01416340 3277. Fax: 416-340-3609.

E-mail address: tereza.martinu@uhn.ca 
bronchial wash; donor bronchoscopy; pediatric bronchoscopy sample inventory/tracking, (8) donor bronchoscopy, and (9) pediatric considerations. This consensus document aims to harmonize clinical and research practices for BAL collection and processing in LTx. The overarching goal is to enhance standardization and multicenter collaboration within the international LTx community and enable improvement and development of new BAL-based diagnostics. J Heart Lung Transplant 2020;39:1171-1190

(C) 2020 International Society for Heart and Lung Transplantation. All rights reserved.

\section{Introduction}

Bronchoscopic bronchoalveolar lavage (BAL) allows sampling of the small airway and alveolar compartment of the lung. In clinical lung transplantation (LTx), BAL is routinely used for monitoring the lung allograft and detecting infections. In research, BAL has been extensively used to improve our understanding of allograft dysfunction and to identify biomarkers with diagnostic and/or prognostic value for phenotyping acute rejection (AR) and chronic lung allograft dysfunction (CLAD). ${ }^{1-5}$ However, comparison of clinical and research data from different institutions, validation of findings, and ultimately clinical applicability are hindered by the high variability of BAL collection and analysis approaches. This constitutes an important barrier for collaborative projects in this setting.

General BAL standardization guidelines were published by the European Respiratory Society (ERS) in 1999, and guidelines specific to patients with interstitial lung diseases were put forth by the American Thoracic Society (ATS) in 2012. ${ }^{6-8}$ Although these guidelines set a great precedent, BAL collection techniques still vary significantly and are often poorly described in the literature. In LTx, specific considerations about BAL collection and processing apply as, in most centers, LTx recipients undergo regularly scheduled surveillance bronchoscopies with BAL sampling; BAL is often performed in the setting of good lung function; and the overall poor outcomes after LTx create a greater mandate for research, patient enrollment, and multicenter collaboration. However, the definitions and techniques used for bronchial and alveolar sampling have never been standardized across LTx centers. The objective of this consensus document, supported by the International Society for Heart and Lung Transplantation (ISHLT), is to assist in standardizing practices across LTx centers around the world by clarifying definitions and techniques and by proposing recommendations for bronchial and alveolar sampling in LTx.

\section{Methods}

The detailed methods used for the creation of this document are presented in Section A of the Supplementary Material, available online at www.jhltonline.org. Briefly, an international workgroup of 66 LTx specialists was created and divided into 9 subgroups covering 9 overarching topics. The subgroups prepared the comprehensive ISHLT BAL survey, capturing BAL collection and processing practices, and administered it to $114 \mathrm{LTx}$ centers from 27 countries. The survey results (Section B of the Supplementary Material online), as well as a systematic literature review (Section $\mathrm{C}$ of the Supplementary Material online), were used for the creation of the statements. All statements were subjected to voting by all workgroup members according to the Delphi method. In the absence of a strong evidentiary base regarding best practices for the acquisition, storage, and processing of BAL fluid, the proposed statements represent consensus recommendations. To avoid repeatedly stating this limitation for most statements, specific grades were used to reflect the level of evidence, as well as strength of agreement within the survey, subgroups, and workforce voting (see grading system in Table 1).

\section{Statements}

\section{Bronchoscopy procedure and BAL collection}

\subsection{Definitions}

The ISHLT BAL survey shows considerable variability in the interpretation of BAL and bronchial wash (BW) definitions and collection techniques among centers around the world; for example, $17.2 \%$ report wedging the bronchoscope when they perform bronchial washings. Major thoracic societies have provided guidance on the performance of BAL, whereas no guidelines exist regarding BW. The ATS Clinical Practice Guideline and the British Thoracic Society guideline recommend that BAL be performed with

\section{Table 1 Grading of the Statements}

Literature level of evidence $^{a}$

A: data derived from multiple RCTs or meta-analyses

B1: data derived from 1 RCT

B2: data derived from large non-randomized studies

C1: data derived from small studies, retrospective studies, or registries

C2: expert opinion, no published data

Strength of survey agreement (S)

S1: excellent: $81 \%-100 \%$

S2: good: $61 \%-80 \%$

S3: moderate: $41 \%-60 \%$

S4: fair: $21 \%-40 \%$

S5: poor: $<20 \%$

Strength of subgroup opinion (0)

OI: strong

OII: moderate

OIII: weak

Strength of workforce agreement based on

Delphi voting (V)

$\%$ of workforce members who voted 8 or 9 out of 9 (i.e., high agreement)

Abbreviation: RCT, randomized controlled trial.

${ }^{\text {a }}$ This scale is in accordance with the grading schema proposed by the International Society for Heart and Lung Transplantation but provides more details for grade $\mathrm{C}$, which was split into $\mathrm{C} 1$ and $\mathrm{C} 2$.

${ }^{\text {bBased on reference }}{ }^{9}$. 
a flexible bronchoscope placed in a wedge position within a selected bronchopulmonary segment. ${ }^{6-8} \mathrm{We}$ agree with these prior published guidelines and propose summary statements below to solidify the definitions. BW has not been previously defined. BAL and BW may yield different information: BAL is considered to sample the alveoli and small airways, whereas BW primarily samples larger airways. If BW is performed in a mainstem or lobar airway, it can be referred to as large airway BW.

BAL is also distinct from what has been termed miniBAL, which samples airways by blind passage of a protected telescoping non-wedged lavage catheter via the endotracheal tube in mechanically ventilated patients. It was first described in $1989 .{ }^{10}$ Among survey respondents, only $27.6 \%$ perform mini-BAL in LTx recipients, and of those, $75.9 \%$ indicate that the procedure is done the same as BAL but with a smaller instillation volume, indicating an important misunderstanding of the term. Therefore, we recommend avoiding the term mini-BAL as specified below.

\section{Statement}

- Bronchoalveolar lavage (BAL) is a method of sampling the lung allograft where sterile isotonic saline is instilled and then aspirated through a flexible bronchoscope, with the tip wedged in a segmental or sub-segmental airway. The instilled volume should be sufficient to reach the alveolar space.

- Bronchial wash (BW) is a method of sampling the lung allograft where the instilled fluid does not reach the alveolar space, due either to low instillation volume or not wedging. BW can be wedged or unwedged.

- Use of the term mini-BAL, which usually refers to a catheter-based BW, is confusing and should be avoided. (C2, S3-5, 0I, V94\%)

\subsection{What are the indications for BAL in LTX?}

The indications for BAL after LTx generally fall into 1 of 2 categories: surveillance or diagnostic. Surveillance refers to a scheduled protocol bronchoscopy in the absence of any clinical suspicion of acute pathology. Diagnostic (or clinically indicated) bronchoscopy is done for suspected pathology such as AR or infection. According to the ISHLT BAL survey, 93.4\% of respondents perform BAL during surveillance bronchoscopy and $91.4 \%$ during diagnostic bronchoscopy. Of note, $34.1 \%$ indicate that large airway BW is performed in addition to or instead of BAL during a surveillance bronchoscopy and $39 \%$ during diagnostic bronchoscopy.

Although broadly utilized, there are no prospective controlled data assessing the utility of surveillance bronchoscopy. In uncontrolled observational studies, surveillance BAL commonly identifies asymptomatic infections (pathogens found in $12 \%-40 \%$ of cases), especially in the first 6 to 12 months post-transplantation ${ }^{11-14}$ and potentially even after the first year. ${ }^{15}$ However, a small single center non-randomized study that compared surveillance with no surveillance showed that, although more infections were diagnosed in the surveillance group, no difference was observed in freedom from CLAD or survival. ${ }^{16}$ In the ISHLT BAL survey, surveillance bronchoscopy is utilized by $86.7 \%$ of the centers. The schedule for surveillance bronchoscopy varies, but most indicate that it is performed at 4 weeks (76.9\%), 3 months (79.1\%), 6 months (73.6\%), and 12 months (80.2\%) post-LTx.

Regarding the indication for diagnostic bronchoscopy, besides suspected infection, surveyed respondents most commonly reported performing it for suspected AR (98.1\%), suspected antibody-mediated rejection (AMR) (81\%), and suspected CLAD (78.1\%). However, apart from ruling out infection, ${ }^{17}$ there are no strong data to show that BAL is useful to diagnose AR, AMR, or CLAD. When BAL is performed for diagnostic reasons, pathogens are identified in $39 \%$ to $69 \%$ of cases. ${ }^{11,12,14,18}$

In a large cohort of LTx patients, BAL was generally well tolerated and safe, whereas serious complications with bronchoscopy were associated with more invasive procedures like transbronchial biopsies. ${ }^{19}$ Specific risk factors need to be considered and include oxygen requirement before bronchoscopy ${ }^{19}$; thrombocytopenia (BAL is considered safe with platelet counts $>20,000$ per $\mu 1^{20}$ ); active cardiac ischemia ${ }^{8}$; and male sex, increase in body mass index after LTx, and presence of obstructive sleep apnea, which increase risk of upper airway obstruction. ${ }^{21}$ Hypoxia often presents post-procedure after the bronchoscope is withdrawn. ${ }^{22}$ Topical lidocaine anesthesia may facilitate minimization of systemic sedation. Although lidocaine can inhibit growth of pathogens in culture, the concentrations of lidocaine measured in BAL fluid are generally well below the reported minimal inhibitory concentrations. ${ }^{23,24}$

Although not directly relevant to BAL standardization, in light of the current severe acute respiratory syndrome coronavirus 2 pandemic and concern over potential future epidemics, we would like to highlight the importance of infection control measures while performing, transporting, processing, and storing BAL. As it is an aerosol-generating procedure, bronchoscopy should only be done with appropriate protection for the operators consistent with current guidelines $^{25,26}$ and as directed by local recommendations. Any of the statements provided herein might be subject to future amendments if compliance with specific biosafety protocols is required per health and safety policies.

\section{Statements}

- BAL is generally well tolerated and safe. Fitness for BAL should incorporate assessment of lung function and comorbidities. (C1, S N/A, OII, V95\%)

- Surveillance BAL at around 1, 3, 6, and 12 months post-transplant is a common and acceptable schedule to identify asymptomatic infection or colonization. (C2, S2, OII, V82\%)

- Diagnostic BAL is useful for the diagnosis or exclusion of respiratory infection. (C1, S2, 0II, V94\%) 


\subsection{What is the optimal technique for performing $B A L$ in LTX recipients?}

As there is still no satisfactory method to determine the dilution factor during lavage, the lack of standardized technique causes great difficulty in interpreting the measurements of BAL components. Previous task force reports and guidelines provide vague guidance, but none are specific to LTx. ${ }^{6-8}$

The route of bronchoscopy is not specified by guidelines. In the ISHLT BAL survey, respondents reported that the oral $(45.7 \%)$ or nasal $(32.4 \%)$ routes for bronchoscopy were preferred over laryngeal mask (11.4\%) and elective intubation (10.5\%). Several respondents noted that they avoid the nasal route in recipients with cystic fibrosis (CF). The middle lobe and lingula result in higher returns of instilled volumes than other lung regions. ${ }^{27}$ Survey respondents confirm that the preferred choice for lavage in bilateral LTx is the middle lobe $(81 \%)$, whereas the lingula was the most common second choice $(72.4 \%)$. Most respondents $(88.6 \%)$ do not perform BAL in more than 1 location during the same procedure. Given the potential for iatrogenic bleeding to alter the cellular and protein components of BAL, it is recommended that BAL be performed before any biopsy or airway brushing, ${ }^{28}$ which is consistent with the approach of most centers surveyed. Several survey respondents comment that suctioning is avoided before BAL and a minority indicate performing a rinse of the bronchoscope channel immediately before wedging for BAL, although prior guidelines have not addressed this technique and there are no supportive data.

Regarding the volume of normal saline to be instilled, prior guidelines have recommended between 60 and $300 \mathrm{ml}$ in total: 100 to $300 \mathrm{ml}$ per ATS, ${ }^{7}>100 \mathrm{ml}$ per ERS, ${ }^{6}$ and 60 to $180 \mathrm{ml}$ per British Thoracic Society. ${ }^{8}$ Studies using $50-$ or $60-\mathrm{ml}$ aliquots demonstrate that the concentration of BAL components in the first aliquot differs from subsequent aliquots. ${ }^{29-31}$ Studies that used two 50 or $60 \mathrm{ml}$ aliquots showed that the cumulative return fluid contained significant concentrations of alveolar-derived proteins, suggesting that this approach is sufficient to achieve lavage of both airways and alveoli, whereas a single 50- to $60-\mathrm{ml}$ aliquot only samples airways. ${ }^{32,33}$ In the ISHLT BAL survey, the most common responses for volumes instilled at a time were $50(28.6 \%)$ or $60 \mathrm{ml}(23.8 \%)$. The mean usual total volume instilled was $100 \mathrm{~mL}$ ( \pm SD 43.8, range 20-200) and the mean maximum total volume instilled was $145 \mathrm{ml}$ ( \pm SD 54.3, range 30-300).

A longer time between fluid instillation and aspiration (i. e., dwell time) results in greater diffusion of molecules from sources other than the epithelial lining fluid into the recovered lavage fluid. ${ }^{34}$ Guidelines consistently recommend immediate aspiration (no dwell time) by low pressure suctioning to avoid airway collapse and to allow maximal retrieval of instilled fluid. Most respondents of the ISHLT BAL survey $(58.1 \%)$ indicate that they aspirate immediately after instillation (i.e., no dwell time). Among the $40 \%$ who preferred a dwell time, there was no consensus on the amount of time waited, ranging from 3 to 30 seconds, or 2 to 3 breaths. Slightly more respondents favor wall (vacuum) over manual suction ( $51.4 \%$ vs $43.8 \%$ ).

Acknowledging the discrepancies in guidelines, literature, and current approaches, we propose an LTx standard protocol based on the most common answers in the ISHLT BAL survey and the data showing that 2 sequential $50-\mathrm{ml}$ aliquots adequately lavage both airways and alveoli. ${ }^{32}$

\section{Statements}

- BAL should be performed by sequentially instilling two 50-ml aliquots (regardless of return volume) in the middle lobe or lingula, unless otherwise directed by abnormal imaging or airway exam. This approach was chosen based on the most common responses from the international BAL survey. (C2, S3, 0I, V86\%)

- Aspiration should be performed immediately (without a dwell time) after instilling each aliquot (i.e., instill $50 \mathrm{ml}$, aspirate, instill $50 \mathrm{ml}$, aspirate). (C1, S3, 0I, V88\%)

- There is no requirement to rinse the bronchoscope channel before BAL. Nevertheless, suctioning before BAL should be avoided when possible. (C2, S2, OI, V79\%)

- Both wall (vacuum) and manual suction methods of aspiration are acceptable, but airway collapse during suctioning should be avoided. (C2, S2, OI, V92\%)

- BAL should be performed before biopsy or airway brushing. (C1, S2, 0I, V96\%)

- Although no specific route is recommended for all bronchoscopies, we recommend avoiding the nasal route in patients with known or potential microbial colonization of the nasopharyngeal airways. (C2, S3, 0II, V73\%)

\section{Sample handling in the bronchoscopy department}

Prior studies have shown that successive BAL return volumes retrieved from the lung have different cellular and protein contents. Rennard et al. ${ }^{35}$ demonstrated that the first returned aliquot was enriched for ciliated airway epithelial cells and contained higher proportions of neutrophils and fewer lymphocytes and macrophages, while being more abundant in content for some large proteins including immunoglobulins A and G. Recently, this has also been shown in LTx patients, suggesting that the first returned BAL aliquot is more representative of the airway compartment, whereas latter aliquots are more representative of the alveolar space. ${ }^{33}$

Consistent with these observations, previous BAL practice guidelines put forth by ERS and ATS have recommended that successive return aliquots be pooled or, at minimum, the sampling method be specified. ${ }^{6,36}$ The results of the ISHLT BAL survey suggest that most of the respondents $(71.4 \%)$ pool successive BAL aliquots into a single sample. Given the current literature and existing practices at LTx centers, we recommend that serial BAL aliquots 
obtained from the same lobe should be pooled before submission for clinical testing. Discarding the first obtained return aliquot was discussed; however, we deemed that this approach would be unlikely uniformly applied to all patients (given different yields of BAL) and would lead to greater discrepancy between patients and centers.

Best practices related to short-term storage of BAL samples in the bronchoscopy area or during transportation to the clinical laboratory have not been rigorously established. The results of the ISHLT BAL survey demonstrated that most of the responding centers hold BAL samples at room temperature in the bronchoscopy area and during transport to the clinical laboratory (73.3\% and 79\%, respectively). The second most common response included holding the BAL sample on ice or placing it in a $4^{\circ} \mathrm{C}$ refrigerator $(21.9 \%$ and $18.1 \%$, respectively). Some highly specific studies, such as aspergillus antigen testing, may require that the sample be held on ice.

\section{Statements}

- Serial BAL aliquots obtained from the same lobe should be pooled before submission for clinical testing. (C1, S2, 0I, V88\%)

- For most clinical purposes, BAL samples can be held at room temperature for short-term storage in the bronchoscopy area and during transportation to the clinical laboratory. However, if storage times are expected to be prolonged (e.g., more than 2 hours), keeping the BAL sample in the refrigerator or on ice is preferred. Delays in sample transport to the clinical laboratory should be avoided as it is likely to impair sample quality. Additionally, when specialized testing is ordered on the BAL fluid or BAL samples are collected for specific research purposes, sample handling should be as recommended by the receiving clinical or research laboratory. (C2, S2, 0II, V90\%)

- Studies reporting results from BAL samples should specify the method of BAL sampling and storage as well as the volume instilled and volume returned. (C2, S N/A, OI, V94\%)

\section{Sample processing and testing for clinical purposes: microbiology}

\subsection{BAL clinical microbiologic studies}

Infection after LTx is common and contributes significantly to morbidity and mortality, especially in the first year after transplant. ${ }^{37-41}$ The ISHLT BAL survey found that $100 \%$ of respondents perform bacterial culture, $87.7 \%$ perform fungal culture, $86 \%$ perform acid-fast bacilli culture, and $70.2 \%$ perform polymerase chain reaction (PCR) for viruses other than cytomegalovirus (CMV) (e.g., influenza, respiratory syncytial virus [RSV], adenovirus, etc.). Over half of centers (59.7\%) reported utilizing CF respiratory bacterial cultures, which employ specific processing and selective media to identify bacterial organisms more commonly identified in patients with
CF. Fewer centers reported routinely performing CMV-specific analysis such as shell-vial assay or PCR (61.4\%), Pneumocystis jirovecii (PJP) testing via either silver stain or PCR (54.4\%), galactomannan (45.6\%), or Nocardia species culture (28.1\%). A small number of centers reported also specifically including Legionella culture. In regard to viral analysis performed, centers reported routinely testing for influenza (80.7\%), RSV (75.4\%), parainfluenza (73.7\%), adenovirus $(73.7 \%)$, rhinovirus $(66.7 \%)$, human metapneumovirus (64.9\%), and herpes simplex virus (HSV) (54.4\%). Of centers performing viral analysis, $82.5 \%$ do so by multiplex PCR. Fewer centers (38.6\%) routinely perform testing for varicella zoster virus.

There is no current consensus or data regarding the appropriate microbiologic studies to perform on BAL collected routinely after LTx. Infectious Disease Society of America (IDSA) guidelines support quantitative cultures of invasively obtained samples in the setting of suspected hospital-acquired pneumonia and ventilator-associated pneumonia. Although quantitative culture of BAL in other settings and populations may be reasonable, the culture thresholds defining pneumonia and/or necessity to treat are not established. PCR-based detection methods are becoming increasingly available, and further studies will be needed to establish their use for infection assessment in LTx patients. Furthermore, endemic infections and pandemic or local epidemic outbreaks of respiratory pathogens may warrant additional specific testing.

\section{Statements}

- The range of infections after LTx is broad; thus, testing of BAL from $L T x$ recipients should include, at the least, bacterial (CF respiratory culture when appropriate), fungal, and mycobacterial cultures, as well as PCR for a range of community-acquired respiratory viruses. (C2, S1, 0I, V87\%)

- Multiplex PCR analysis for respiratory viruses should include influenza, RSV, parainfluenza, adenovirus, rhinovirus, and human metapneumovirus. Centers may also consider testing for bocavirus and/or coronavirus. Analysis for HSV or varicella zoster virus may be considered when clinically appropriate. (C2, S2, 0II, V85\%)

- CMV-specific analysis, PJP testing, galactomannan, and culture for Nocardia species should be sent when clinically appropriate. (C2, S3, 0II, V88\%)

\subsection{Laboratory processing of BAL samples in the microbiology lab for clinical purposes}

No data exist in regard to the recommended laboratory processing of BAL samples in the microbiology laboratory, specifically for samples collected from LTx recipients, and most laboratories devise their own individual standard operating procedures. IDSA and the American Society for Microbiology published a joint document offering some 
guidance regarding diagnostic procedures and sample transportation, recommending that BAL fluid be placed into a sterile container that may be maintained at room temperature for up to 2 hours or in a $4^{\circ} \mathrm{C}$ refrigerator up to 24 hours after collection. ${ }^{42}$ The ISHLT BAL survey found that $66.7 \%$ of centers store BAL fluid at room temperature before processing and $38.6 \%$ in a $4^{\circ} \mathrm{C}$ refrigerator. Centers reported a maximal acceptable delay of 6 hours $(45.6 \%)$ or other $(26.3 \%)$ with comments indicating that acceptable delay in processing depends on the testing ordered.

The IDSA/American Society for Microbiology guideline does not comment on the minimum quantity needed for individual microbiologic analyses. Respondents to the ISHLT BAL survey reported a minimum quantity needed for standard post-transplant-related microbiologic analysis to be $10.9 \pm 8.5 \mathrm{ml}$. The largest proportion of centers reported a minimum quantity of BAL fluid to be $10 \mathrm{ml}$ (26.3\%), whereas almost an equal number reported a minimum quantity of $5 \mathrm{ml}(24.6 \%)$. Most centers do not mention BAL sample quality in their clinical reporting $(63.2 \%)$, whereas $22.8 \%$ will comment only when BAL quality is low.

Approximately half (50.9\%) of centers reported that centrifugation of BAL samples before further processing was not needed, whereas $29.8 \%$ reported that centrifugation should be performed. If centrifugation occurs, centers

\section{Statements}

Note: Centers may be constrained by their individual laboratory standard operating procedures. Although this document does not propose standard operating procedures for processing of BAL samples in the microbiology lab, we support such an endeavor as an important longer-term goal and make the following general recommendations:

- BAL fluid should be placed into a sterile container that may be maintained at room temperature for up to 2 hours or in a $4^{\circ} \mathrm{C}$ refrigerator up to 24 hours after collection. (C1, S2, 0I, V94\%)

- The minimum quantity needed for standard posttransplant-related microbiologic analysis may depend on the requirements of an individual center's microbiology lab; we recommend $10 \mathrm{ml}$, but a range of 5 to $15 \mathrm{ml}$ is reasonable. (C2, S2, 0I, V88\%)

- Reporting on BAL quality is likely not needed but should be considered if there is concern that the quality is low and may impact the testing results. If quality is reported, specific factors to consider are excessive red blood cells, low return of instilled volume, lack of alveolar macrophages possibly indicating absence of alveolar sampling, excessive epithelial cells indicating airway sampling contamination, or other degenerative factors of note. (C2, S3, OII, V92\%)

- Centrifugation is generally not needed but may be required for certain tests or by a center's individual laboratory practices. (C2, S3, 0II, V82\%)
Table 2 Minimum BAL Sample Information Required for the Microbiology, Cytology, and Research Laboratories

\begin{tabular}{|c|c|c|c|}
\hline Variable & Micro lab & Cytology lab & Research lab \\
\hline $\begin{array}{l}\text { Identifying infor- } \\
\text { mation (name, } \\
\text { medical record } \\
\text { number, date of } \\
\text { birth) }\end{array}$ & Yes & Yes & - \\
\hline $\begin{array}{l}\text { Patient/sample } \\
\text { research ID }\end{array}$ & - & - & Yes \\
\hline Patient consent & - & - & Yes \\
\hline $\begin{array}{l}\text { Lung transplant } \\
\text { status }\end{array}$ & Yes & Yes & Yes \\
\hline $\begin{array}{l}\text { General clinical } \\
\text { information }\end{array}$ & Yes & Yes & - \\
\hline Tests requested & Yes & Yes & - \\
\hline Sex & - & - & Yes \\
\hline Age & - & - & Yes \\
\hline Native lung disease & - & - & Yes \\
\hline Ethnicity & - & - & Yes \\
\hline Date of transplant & - & Yes & Yes \\
\hline Type of transplant & - & - & Yes \\
\hline $\begin{array}{l}\text { Date of } \\
\text { bronchoscopy }\end{array}$ & Yes & Yes & Yes \\
\hline $\begin{array}{l}\text { Indication for } \\
\text { bronchoscopy }\end{array}$ & Yes & Yes & Yes \\
\hline $\begin{array}{l}\text { Performed } \\
\text { procedures }\end{array}$ & - & - & Yes \\
\hline Location of BAL & Yes & - & Yes \\
\hline Volume instilled & - & - & Yes \\
\hline Return volume & - & - & Yes \\
\hline
\end{tabular}

Abbreviations: BAL, bronchoalveolar lavage; ID, identifier; Micro, microbiology.

reported a median (range) of 10 (5-20) minutes at a speed of $1,750(250-3,000)$ relative centrifugal force $(\mathrm{rcf}) / \mathrm{g}$ or $1,500(1,000-3,000)$ revolutions per minute (rpm).

The minimum clinical information required to facilitate proper processing in the microbiology laboratory should include patient identifiers, status as a LTx recipient, relevant clinical history, and testing required, as outlined in Table 2.

\section{Sample processing and testing for clinical purposes: cytology}

\subsection{BAL clinical cytological and cell count studies}

Microscopic cytology examination: Of the 105 ISHLT BAL survey participants, $61 \%$ reported that they routinely request cytology with pathology review during each surveillance bronchoscopy; $22.9 \%$ request cytology evaluation for suspected infection, $13.3 \%$ for suspected rejection, and $35.2 \%$ for suspected malignancy, and $7.7 \%$ stated they never request cytology evaluation for post-transplant bronchoscopies. Although commonly requested in routine lung recipient care, the value of sending BAL cytology with pathology examination as a routine study has been questioned, particularly in light of the relative cost. ${ }^{43,44}$ Prior 
studies examining the diagnostic performance of BAL cytology for infection have yielded conflicting results. $\mathrm{Al}$ Zaabi et al. ${ }^{43}$ demonstrated a poor detection rate for infectious agents utilizing cytology. In contrast, a study by Walts et al. ${ }^{45}$ in 1991 showed good diagnostic capacity of cytology for non-bacterial organisms-specifically Candida species, although this is often not a pulmonary pathogen-and HSV. Additionally, special staining of cytology specimens may be a useful adjunct for the identification of difficult-toculture organisms, such as Mucor or Nocardia species, or in cases of suspected PJP.

Beyond detection of infection, another common application of BAL cytology examination is in the detection of malignancy, particularly relevant in the immunocompromised LTx population. A small study by Ohori et al. ${ }^{46}$ examining atypical epithelial cells from BAL fluid of LTx recipients compared with those from non-transplant patients with known lung carcinoma determined that the evaluation of cytological features alone may not permit differentiation of atypical cells in non-neoplastic conditions within the lung recipient from those in malignant conditions. In addition, detection of lipid-laden macrophages may indicate chronic aspiration or gastro-esophageal reflux. ${ }^{47}$

Cell counts: Examination of the cellular composition of the BAL fluid and the correlation of BAL cell populations with acute and chronic rejection in particular has been an intense area of research interest in the LTx community. In the ISHLT BAL survey, $71.4 \%$ of respondents reported that BAL differential cell counts are performed routinely on their post-transplant bronchoscopies. Among 54 cytology labs surveyed, $14.8 \%$ stated that they performed cell counts and/or differentials only, $18.5 \%$ performed microscopic examination only, and most labs (53.7\%) combine cell counts and/or differentials with microscopic examination.

The literature indicates that cytological findings on BAL do not adequately distinguish between $\mathrm{AR}$ and infection. $^{48,49}$ Although the cytological changes on BAL (an early lymphocytosis followed by a rise in neutrophils within the fluid) cannot be considered specific for AR, they do raise clinical suspicion. ${ }^{49,50}$ With regard to the utility of BAL cell counts to aid in the detection of CLAD, as summarized in a recent review, ${ }^{1}$ several studies have now demonstrated a significant association between BAL neutrophilia and concurrent or future CLAD, with the significant neutrophil percentage cut-off identified at $16 \%$ to $24 \% .^{49,51-53}$ Significant $(>2 \%)$ eosinophilia in BAL was also associated with lower overall and CLAD-free survival. ${ }^{54}$ BAL eosinophilia may further associate with worse outcomes specifically after diagnosis of restrictive allograft syndrome. ${ }^{54,55}$ Although clear risk thresholds have not been established in multicenter studies, clinical examination of the BAL differential inflammatory cell count may provide useful information in the assessment of patients with loss of lung function.

There is no good evidence to support the best approach to determining BAL cell counts. Most centers mention using at least 1 approach for BAL cell quantification: cell count $(65.7 \%)$, differential $(71.4 \%)$, and/or microscopic analysis $(61.0 \%)$. With respect to the inclusion of epithelial cells in the differential, the literature was not particularly revealing, as the vast majority do not provide this information (95\%). Only 2 papers mention the inclusion of epithelial cells in the differential, ${ }^{43,56}$ which may be important in (1) assessing the representativeness of the specimen, (2) detecting potential cytopathogenic effects of viral infections and (3) uncovering signs of epithelial malignancy.

\section{Statements}

- There is insufficient evidence to recommend routine morphological microscopic cytology for the detection of infection, malignancy, or rejection as standard practice in all clinical post-transplant bronchoscopies. Microscopic cytology may, however, be of clinical benefit in cases where the clinical suspicion for atypical infection or malignancy is high. (C1, S4, 0II, V92\%)

- All post-transplant BAL samples should include a differential cell count with or without an absolute cell count (using an automated cell counter or manual cell counting approach). (C2, S3, 0I, V92\%)

- When assessing loss of lung function, analysis of the BAL differential cell count may be a useful aid in narrowing the differential diagnosis. (C1, S2, OII, V91\%)

- At present, there is insufficient evidence for the inclusion of epithelial cells in the differential BAL cell count for diagnostic purposes. However, quantification of epithelial cells can provide information about representativeness and overall quality of the BAL sample. (C2, S3, 0II, V89\%)

\subsection{Techniques for cytological studies of $B A L$ samples}

With respect to the minimum BAL volume required for adequate cytological assessment, according to the survey respondents, $1 \mathrm{ml}(16.7 \%), 5 \mathrm{ml}(35.2 \%)$, or $10 \mathrm{ml}(18.5 \%)$ would suffice for a standard cytological analysis of BAL, with only a minority using more than $10 \mathrm{ml}$. Only a few papers comment on this, suggesting that 15 to $30 \mathrm{ml}$ is sufficient for a full analysis. ${ }^{57-61}$

In the relevant literature, volumes of BAL instilled or retrieved for cytological analysis are mentioned in a minority of papers. Bollmann et al. ${ }^{62}$ found that, although significantly larger volumes were returned with a $5 \times 20 \mathrm{ml}$ instillation protocol, cellular concentration was higher when using a $2 \times 50 \mathrm{ml}$ protocol. The latter regimen, therefore, may be preferable for cytological diagnosis and is consistent with the recommended BAL collection outlined in Section 1 of this document.

Once received by the laboratory, most survey respondents $(53.7 \%)$ centrifuge their samples. Among those who mention the use of centrifugation, the median (range) of time is $10(3-20)$ minutes and the median (range) of speed 
$390(72-616) \mathrm{rcf} / \mathrm{g}$ or 2,000 (800-4,400) rpm. Most papers mention the use of centrifugation, but with a wide range of conditions: median speed of 400 to $500 \mathrm{~g}$ (range, $40-1,000 \mathrm{~g}$ ) and median duration 5 to 10 minutes (range, 2-20 minutes) (Section C of the Supplementary Material online). Of 140 papers that describe centrifuge methodology, 36 (26\%) mentioned the use of the cytospin technique.

Only a minority of ISHLT BAL survey respondents employ red cell lysis (14.8\%). The literature assessed does not provide significant insight in this matter, as only 7 papers comment on the use of red cell lysis, of which 6 use this technique ${ }^{4,50,63-66}$ and 1 does not. $^{67}$

For morphological assessment, most laboratories use a hematoxylin and eosin (H\&E) (42.6\%) or H\&E equivalent stain $(9.3 \%)$ and/or Papanicolaou stain $(40.7 \%)$, with Giemsa stains $(20.4 \%)$ also being commonly employed. In addition, fungal stains are frequently used $(33.3 \%)$. Of the assessed papers, most $(69 \%)$ do not comment on the stains used, although most of those that do employ a Giemsa stain in isolation (18\%) or a combination of stains (e.g., Giemsa + Papanicolaou stain, with or without fungal or iron stains).

The initial step in morphological evaluation of the BAL specimen could be an assessment of the suitability of the specimen received. However, only $24.1 \%$ of laboratories surveyed comment on the quality of the BAL specimen. With regard to the literature, very few papers comment on the need for reporting the quality of BAL specimens for cytology (6.9\%).

The minimum clinical information required to facilitate proper processing in the cytology laboratory is summarized in Table 2.

\section{Statements}

- Ideally, $10 \mathrm{ml}$ of recovered lavage fluid should be sent for cytology, with a minimum of $5 \mathrm{ml}$; less than $5 \mathrm{ml}$ is usually inadequate for a reliable assessment of cytology. (C2, S3, OI, V84\%)

- Centrifugation is not required for cytological analysis of BAL fluid. However, if separation of cell pellet from supernatant is desired, we recommend centrifugation at 400 to $500 \mathrm{~g}$ for 5 to 10 minutes and a second centrifugation of the resuspended pellet to produce cytospin slides according to the device instructions. (C2, S3, 0II, V89\%)

- There is currently insufficient evidence for or against the use of red cell lysis before cytological analysis of BAL fluid. (C2, S2, 0II, V92\%)

- Basic cytomorphological analysis of the BAL fluid should be performed using routine stains, employing (a combination of) H\&E (or equivalent), Giemsa, or Papanicolaou stains. Additional special stains (e.g., fungal and iron stains) may be performed based on clinical and cytomorphological suspicion. (C2, S1, 0I, V87\%)

- It is advisable to comment on the quality of the BAL specimen for cytology, in line with recommendations for cytology reporting in general. (C2, S4, 0II, V85\%)

\section{BAL sample processing and testing for research}

\section{Introduction: What can and is being done with BAL fluid for research purposes?}

BAL-focused research is an invaluable investigational tool in LTx and should be done with proper institutional review board approval and patient consent. Informed consent can be obtained for specific studies or an open consent can be used to support bio-banking for future research. Cells, proteins, and other components identified in BAL can provide essential insights into LTx biology and diagnostic information regarding allograft infection or rejection. As examples, BAL biomarkers of club cells, aspiration of gastric content, and mesenchymal progenitor cells have identified biological processes relevant to CLAD development, ${ }^{68-70}$ whereas scores based on immunophenotyping of BAL cells have quantified AR, infection, and CLAD risk. ${ }^{4}$

Although many concepts are relevant to all BAL projects in LTx research, detailed approaches need to be tailored to specific questions. Our literature review identified a wide variety of target analytes, measured in the BAL either as part of clinical care $(57.3 \%)$ or as part of a research study (49.1): $51.2 \%$ assessed microbiology, $3.1 \%$ evaluated the microbiome, $43.8 \%$ assessed cells, $32.2 \%$ focused on proteins, $18.3 \%$ DNA or RNA, and $23.4 \%$ included other analytic targets.

Based on the ISHLT BAL survey, $57.1 \%$ of centers were using BAL for research. Of the 42 centers that completed the research-specific survey section, $57.1 \%$ collected and banked BAL samples, whereas $42.9 \%$ collected samples for specific research projects without active bio-banking. Regarding sample types, $47.6 \%$ collected raw BAL, $69 \%$ supernatant, $64.3 \%$ cell pellets, and $11.9 \%$ BW samples. Although $61.9 \%$ of centers had no specific analyte planned at the time of sample collection, $40.5 \%$ were performing leukocyte phenotyping and microbiome analyses, 35.7\% protein analyses, and 31\% RNA expression studies (other end points listed in the Supplementary Material S4Q11 online).

\subsection{How should BAL for research be done?}

One major limitation in the literature is inadequate reporting on the details of the BAL collection procedure; for example, most studies do not report on instillation volume and aliquots, location of sampling, and processing of the BAL (Table 3).

Based on the ISHLT BAL survey, of the centers which perform BAL-based research, $65 \%$ reported not changing the BAL procedure or the total instilled volume for research. When planning to use BAL for research in addition to the clinical purposes, $26 \%$ of the centers increase the instilled volume. Among respondents who change the instilled BAL volume for research, there is a wide range of instilled BAL volume (60-200 ml; mean, $137.2 \mathrm{ml}$; SD, 39.3).

There is no universally accepted protocol for the volume or the number of instilled aliquots for the optimal BAL 
Table 3 Bronchoscopy and BAL Details Reported Previously and Recommended for Future Reporting in BAL-Focused Papers

\begin{tabular}{|c|c|c|}
\hline Variable & $\begin{array}{c}\text { Reported in } \\
\text { previous papers }\end{array}$ & $\begin{array}{l}\text { Recommended minimum } \\
\text { required information }\end{array}$ \\
\hline \multicolumn{3}{|l|}{ Bronchoscopy } \\
\hline Time post-transplant & $61.3 \%$ & Yes \\
\hline Route of bronchoscopy & $14.3 \%$ & - \\
\hline Other procedures & $48.4 \%$ & Yes \\
\hline Sequence of procedures & $16 \%$ & - \\
\hline \multicolumn{3}{|l|}{ Sample collection procedure } \\
\hline Rinsing of bronchoscope & $1.7 \%$ & - \\
\hline Location of sampling & $42.1 \%$ & Yes \\
\hline Volume instilled & $51.8 \%$ & Yes \\
\hline Number of aliquots & $40.4 \%$ & Yes \\
\hline Dwell time & $5.5 \%$ & - \\
\hline Information on suction & $16 \%$ & - \\
\hline \multicolumn{3}{|l|}{ Sample storage and processing after collection } \\
\hline Pooling of BAL & $25.1 \%$ & Yes \\
\hline Storage between collection and processing & $24.2 \%$ & Yes \\
\hline Storage of unprocessed (raw) BAL & $11 \%$ & Yes \\
\hline BAL cell pellet preservation & $22.9 \%$ & Yes \\
\hline BAL supernatant processing & $25 \%$ & Yes \\
\hline Centrifugation conditions & $29 \%$ & Yes \\
\hline Processing of sample in lab & $42.7 \%$ & Yes \\
\hline Storage after processing & $23.4 \%$ & Yes \\
\hline Quality assessment & $4.2 \%$ & Yes \\
\hline Normalization of sample & $4.4 \%$ & Yes \\
\hline
\end{tabular}

Abbreviation: BAL, bronchoalveolar lavage.

return for research purposes. Although the practice of using multiple instilled aliquots to reach a total volume $\geq 100 \mathrm{ml}$ has been recommended ${ }^{71}$ and used in a number of studies, ${ }^{4,62,72}$ uncertainty remains as to how variation in the instilled volume can affect the measurements of analytes in BAL. Indeed, decreased BAL fluid return volume has been associated with infection and rejection in LTx recipients. ${ }^{73}$ In a study comparing 2 sequential $50 \mathrm{ml}$ lavages, the first lavage was enriched with neutrophils, airway epithelial cells, and their secreted proteins, whereas the second lavage had higher cell viability and alveolar surfactant protein D. ${ }^{33}$ At a fixed total volume, the number of instilled aliquots may also affect BAL analytes, as one study observed that instilling five $20-\mathrm{ml}$ aliquots resulted in higher BAL return but lower median cell count than using 2 aliquots of $50 \mathrm{ml}^{62}$

\section{Statements}

- Research on BAL fluid from consented LTx recipients is important to understanding post-transplant processes and working toward improving long-term outcomes for this population. (B2, S1, 0I, V94\%)

- We recommend performing the BAL collection per clinical protocol described in Section 1, without specific modifications for research when feasible. However, if BAL collection needs to be modified, we recommend that the collection methodology be consistent for all subjects within a study (C2, S N/A, OII). Collection of additional BAL fluid to a total instilled volume of $\leq 200 \mathrm{ml}$ does not present a significant risk for appropriately selected research participants post-transplant. However, modifications of the BAL collection technique may affect results. ( $\mathrm{C} 1$, S2, 0I, V94\%)

- The BAL collection methodology should be reported in detail for research studies. In addition to the parameters outlined in Section 7 and in Table 2, we recommend reporting on quality controls and $\mathrm{BAL}$ normalization, if done. (C2, S3, OI, V91\%)

- Acknowledging that useful information can be gained despite differences in BAL procedures, we recommend that investigators attempt to ensure consistency in BAL collection techniques across multicenter study sites whenever possible. (C2, S N/ A, 0I, V97\%)

\subsection{What are common practices for handling collected BAL for research?}

In the ISHLT survey of centers performing BAL for research, there was substantial variability in techniques. 
Before arrival to the research lab, $35.7 \%$ of centers kept samples at room temperature, $50 \%$ placed samples on ice, and $21.4 \%$ froze samples either at $-20^{\circ} \mathrm{C}$ or $-80^{\circ} \mathrm{C}$. Although $75 \%$ of centers processed samples within 6 hours, with some reporting significant loss in cell viability at 3 hours, $7.1 \%$ considered a delay of 24 hours before sample processing acceptable.

Filtration of BAL was also highly variable between centers. About $19 \%$ of respondents filtered raw BAL though gauze before storage or centrifugation, whereas many centers used no filtering, and a few used cell separation mesh filters (of varied opening size). Although filtration may be particularly important for flow cytometry (to minimize clogging of the cytometer nozzle), it may not be necessary for other techniques. There is also a concern that filtration could affect results by selectively binding cells or proteins, although the evidence for this is sparse. Similarly, for centrifugation, centers used a range of speeds and times, depending on the target analyte.

A wide range of sample aliquot volumes is stored at different centers. BAL cell pellets are stored alternatively in phenol, TRIzol/QIAzol, RNAlater, Allprotect, DNA/RNA Shield, saline, dimethyl sulfoxide in fetal calf serum, RPMI in fetal calf serum, or RLT buffer. At appropriate concentrations, glutaraldehyde- and formalin-containing storage buffers can inactivate viral pathogens. ${ }^{74}$ BAL cell pellets, supernatant, and raw fluid are most commonly stored at $-80^{\circ} \mathrm{C}$, although some centers use $-20^{\circ} \mathrm{C}$ and others stored in liquid nitrogen.

\section{Statements}

The recommended approach to processing and storing BAL depends on the intended analyses:

- Generally, BAL should be kept at $4^{\circ} \mathrm{C}$ and processed within 24 hours or processed within 2 hours if at room temperature (statement 2.2). (C2, S2, OII, V $97 \%$ )

- For cellular analyses, we recommend centrifugation at $250 \mathrm{~g}$ for 10 minutes, as higher speeds may not be optimal for preserving viable cells (statement 4.2.2). The BAL pellet can be resuspended and cryopreserved in a $10 \%$ final concentration of dimethyl sulfoxide in fetal calf serum ${ }^{75}\left(C_{1}\right.$, S2, OI, V92\%). Centrifugation forces (in $\mathrm{g}$ or $\mathrm{rcf}$ ) should be reported rather than speeds (rpm) because rpm corresponds to different centrifugal forces based on the rotor size.

- Filtration is recommended if mucus or debris could affect the analysis, including flow cytometry analysis of cells. Filtration may result in selective binding and loss of proteins or cells. (C2, S2, OII, V91\%)

- Treatment of cell pellet or neat fluid with RNA stabilization buffers (e.g., TRIzol/QIAzol, RNAlater, Allprotect, and DNA/RNA Shield) and rapid freezing is recommended for analysis of RNA. ${ }^{76}$ TRIzol can be useful for analyzing microRNA but may impede extraction of DNA. (C1, S N/A, OI, V88\%)

- Exosomes can be isolated from cell-free BAL fluid using an ultracentrifugation protocol. ${ }^{77}$ (C2, S N/A, OI, V85\%)

- For protein analysis, we recommend BAL be kept on ice before centrifugation at $650 \mathrm{~g}$ for 10 minutes. A second spin may help reduce residual cellular material. (C2, S3, OII, V87\%)

- Cryostorage at $-80^{\circ} \mathrm{C}$ is adequate for RNA, DNA, and protein, ${ }^{78}$ but liquid nitrogen storage temperatures are recommended for later assays of cellular function. (C2, S N/A, 0II, V88\%)

\subsection{What are the recommendations for normalization of BAL analytes, storage, and quality control?}

Variation in BAL collection techniques can affect analyte concentrations, and there is no universally accepted method for normalization of BAL analyte concentration. Of centers collecting BAL for research, $23.8 \%$ reported not normalizing BAL analytes. The most common normalization parameter was the return volume of BAL fluid collected $(16.7 \%)$, whereas some centers reported using total protein, albumin concentration, or plasma to BAL albumin or urea ratios. Normalization can have unpredictable effects on results.

The most frequently recommended quality metric in the survey was the time between collection and processing, although some centers recommended the percentage of epithelial cells, quantity of mucous, or protein or albumin concentrations. One fourth of centers would discard samples that have passed a threshold time (between 2 hours and 7 days) from collection to processing, $7.1 \%$ of centers would discard samples because of a high percentage of epithelial cells, and $2.4 \%$ because of high quantities of mucous.

\section{Statements}

- Normalization methods are variable and can substantially impact results. If analytes are normalized, non-normalized data should also be provided. (C2, S $\mathrm{N} / \mathrm{A}, \mathrm{II}, \mathrm{V} 90 \%$ )

- Normalization is not always appropriate. If done, careful consideration should be made as to the effects of disease states on the normalization parameters. (C2, S N/A, OIII, V90\%)

- There are no specific thresholds to discard BAL for research, but we recommend documentation of the quality metrics. (C2, S N/A, OI, V88\%) 


\section{BAL microbiome analysis for research}

\subsection{Are there special considerations for $B A L$ collection and processing, relating to potential downstream use for microbiome studies?}

There has been growing interest in the potential impact of the microbiome in LTx over the past decade. ${ }^{79-94}$ Of 42 centers responding to the survey, $17(38.1 \%)$ reported analysis of the bacterial microbiome, with fewer indicating analysis of viral and fungal microbiomes $(26.2 \%$ and $28.6 \%$, respectively). However, there are to date only a limited number of published microbiome studies conducted using BAL fluid samples and very few addressing or comparing technical aspects of BAL specimen collection or handling.

Although a few studies advocate use of special techniques during bronchoscopy (e.g., double bronchoscopy, laryngeal mask airway, and endotracheal tube) to attempt to minimize oropharyngeal contamination during the collection of BAL for microbiome analysis, ${ }^{95}$ these may be impractical for use in routine clinical practice. In view of the often low microbial burdens present in BAL samples and the corresponding potential for confounding by high relative abundance of environmental contaminants, ${ }^{88,96}$ the subgroup consensus view was that concurrent analysis of negative control samples collected before bronchoscopy should be considered. Further, although personal protective equipment (e.g., gloves, gowns, and masks) are routinely used during bronchoscopic procedures, similar precautions should be taken during subsequent specimen handling and processing to minimize the risk of contamination by microbiota from the user. Regarding the storage of samples between collection and processing, a review of both the transplant and non-transplant BAL microbiome literature found that specimens were placed on ice or at $4^{\circ} \mathrm{C}$ before transfer in the large majority of studies but with variability in reporting the delay between procurement and processing/ analysis.

\section{Statements}

- For microbiome analysis, bronchoscopy and BAL fluid collection should be performed as recommended in Section 1. Where feasible, use of special techniques to minimize oropharyngeal contamination for microbiome analysis may be applied, and personal protective measures (e.g., gloves and masks) should be used when handling and processing specimens to minimize contamination from the user. (C2, S N/A, OI-II, V96\%)

- Where participation in microbiome studies is being considered, the most rigorous approach includes collection of control specimens for each bronchoscopy procedure, consisting of 10 to $20 \mathrm{ml}$ of each of the following: blank fluid to be used for the BAL, blank fluid aspirated through the bronchoscope suction channel before insertion into the patient, and oral rinse (mouth wash) sample using fluid from the same batch of fluid to be used for the BAL. (C1, S N/ A, OI, V88\%)

- Where participation in microbiome studies is being considered, BAL specimens should be kept at room temperature for no more than 2 hours and at $4^{\circ} \mathrm{C}$ for no more than 24 hours before processing and/or freezing for longer-term storage. (C2, S1, OI-II, V91\%)

\subsection{How should BAL for microbiome analysis be fractionated/processed before storage, if at all?}

There is a diversity of opinion and limited evidence regarding the need for, relative advantages or disadvantages of, and techniques of fractionation of BAL before storage and downstream microbiome analysis. In its discussions, the subgroup recognized the range of practice and the perceived relative merits and disadvantages of each specimen type. It was acknowledged that raw vs fractionated specimens might be preferable in different situations, depending on the type of microbiome analysis performed, bearing in mind that BAL supernatant may be suboptimal for some types of microbiome analysis. ${ }^{88}$

\section{Statements}

- Fractionation of BAL into cellular (cell pellets) and acellular (supernatant) fractions may be performed based on institutional priorities for types of microbiome analysis (e.g., bacteriome vs virome, cellassociated bacteria vs cell-free bacteria, etc.). In such instances, details of protocols used should be recorded. (C2, S1, 0II, V90\%)

- More data are needed regarding the impacts of different fractionation protocols on microbiome analyses. (C2, S N/A, OI, V93\%)

\subsection{How should BAL fluids be stored for later microbiome analysis?}

In review of the BAL microbiome literature, the vast majority of both transplant and non-transplant studies report storage at $-80^{\circ} \mathrm{C}$ before use for microbiome analysis, with very few reporting storage of cell pellets in RNAlater or other preservation agents. However, the effects of such preservation reagents on downstream microbiome analysis have not been well characterized and require further investigation.

The working group concluded that whereas 1 to $2 \mathrm{ml}$ of BAL fluid may suffice for bacteriome analysis, studies of the mycobiome or virome may require larger volumes. 


\section{Statements}

- Where participation in microbiome studies is being considered, long-term storage of BAL fluid, cell pellets, and/or supernatants should be at temperatures of $-80^{\circ} \mathrm{C}$ or lower. (B2, S1, OI-II, V90\%)

- Where microbiome analysis is desired, a minimum of $2 \mathrm{ml}$ of BAL (raw and/or fractionated) should be stored, with higher volumes recommended $(10 \mathrm{ml}$ or more) if virome analysis is to be performed. (C2, S N/ A, 0I, V82\%)

- The effects of preservation agents (e.g., RNAlater) on downstream microbiome analysis have not been well characterized and require further investigation. (C2, S N/A, OI-II, V88\%)

\section{Sample inventory/tracking and linkage among clinical and research samples and clinical data}

\subsection{What is the minimum information accompanying bio-banked/research samples?}

In the literature, the guidelines on the use of BAL in interstitial lung disease ${ }^{7}$ do not make specific recommendations for annotation or tracking of BAL samples for clinical or research purposes. Based on the ISHLT BAL survey, most LTx bronchoscopists believe that samples should be deidentified and accompanied by data that includes sex, age, native lung disease, date of transplant, type of transplant, date of bronchoscopy, indication for bronchoscopy, and a description of the procedures performed (Table 2).

\section{Statement}

- Research BAL samples should be de-identified and accompanied by data that include sex, age, native lung disease, date of transplant, type of transplant, date of bronchoscopy, indication for bronchoscopy, and a description of the procedures performed. The location of the BAL, the volume instilled, and the volume retrieved should also be recorded (Table 2). (C2, S2, 0I, V97\%)

\subsection{How should samples be labeled and tracked for research purposes?}

There is no literature available regarding labeling or tracking of research BAL samples. The ISHLT BAL survey indicated that only a unique sample ID is crucial to enable database linkage; however, the date and type of samples are also considered relevant information.

\section{Statement}

- Research samples should be labeled with a unique identifier to enable database linkage. Study funding should support adequate infrastructure and dedicated personnel for BAL bio-banking and data retention. Data can be retained in a secure spreadsheet, local laboratory information management system database, or (particularly for multicenter studies) a secure cloud-based platform. (C2, S2, 0I, V93\%)

\subsection{What are key pieces of information about the $B A L$ collection that need to be reported in research manuscripts?}

Surprisingly, the literature search showed that $26 \%$ of BALfocused articles contained no details concerning the bronchoscopy procedure, $44 \%$ had no information about the sample collection procedure, and $33 \%$ included no information about sample processing. Table 3 summarizes the frequency of detail reporting in prior BAL-focused research papers, related to the bronchoscopy procedure itself, sample collection, and subsequent sample processing. The minimal required information, as identified by most of the ISHLT BAL survey respondents, is indicated.

\section{Statement}

- Published studies should report, at a minimum, whether the procedure was conducted according to this ISHLT Consensus Statement, timing post-transplant, route of bronchoscopy, description of other procedures accompanying the BAL, BAL location(s), the volume instilled, the number of aliquots used, the pooling procedure, storage conditions between collection and processing, how the sample was processed in the lab (specifically regarding BAL cell pellet and supernatant separation and centrifugation conditions), details of preservation solutions, and subsequent long-term storage conditions (Table 3). (C2, S3, 0I, V97\%)

\section{Donor bronchoscopy}

\subsection{What constitutes the minimum assessment of the donor airways?}

Among U.S. regulatory agencies and organ procurement organizations, minimal donor assessment guidelines for donor lung evaluation are not uniform (Association of Organ Procurement Organizations policy CL4.E.5.3 and Organ Procurement and Transplantation Network/United Network of Organ Sharing policy 2.11.D). Bronchoscopy 
in donor organ assessment can provide information that may not be readily available on chest radiographs or manual inspection. ${ }^{97,98}$ Fiberoptic bronchoscopy of donors may maximize organ utilization through airway clearance, anatomical assessment, and identification of infectious organisms. $^{99-101}$ The ISHLT BAL survey showed that most (72.4\%) perform bronchoscopy for culture analysis. Bronchoscopy and sample collection in donor assessment is uniformly done before procurement and varies from several days before to intraoperatively during the procurement ${ }^{98,102}$ (Association of Organ Procurement Organizations policy and Organ Procurement and Transplantation Network/ United Network of Organ Sharing policy). Evidence for the specific timing of airway sample collection is lacking.

\section{Statement}

- Fiberoptic bronchoscopy must be performed as part of the lung donor evaluation before procurement to optimize organ function, identify anatomical abnormalities, assess for evidence of infection or aspiration of gastric contents, and obtain a culture for directed antibiotic therapy. (C2, S2, 0I, V95\%)

\subsection{How should sampling from donor airways be performed?}

8.2.1. Approach to donor airway sampling for clinical purposes. Reports on airway sampling for microbiological assessment of potential organ donors have included tracheal aspirates, BW, and BAL. ${ }^{97,99,100,103-106}$ Precise explanation of the sampling methodology is rarely available, making evidence-based practice recommendations problematic. The ISHLT BAL survey demonstrated significant variation in donor airway sampling methodology and technique; however, bronchoscopic aspiration of secretions (35.2\%) and large airway BW (28.6\%) were by far the most common practices. Additionally, $8.5 \%$ of centers perform large airway swabs, $6.7 \%$ perform a low-volume BAL or BAL different than that done in the recipient, and only $4.8 \%$ do a

\section{Statement}

- There is limited evidence and wide practice variation regarding airway sampling technique, site, side, or volume for clinical donor lung assessment. Recommendations from this expert panel, based on common practices within the LTx community, support an unwedged (or wedged) low-volume $(20 \mathrm{ml})$ BW from a site of radiological concern or, if normal, a default location of middle lobe or lingula for infectious risk assessment. Reporting should include whether the scope was wedged or unwedged, the volume of fluid instilled, and the location. (C1, S5, 0I, V94\%) standard BAL similar to what would be performed in the recipient. Complicating this observation is the limited evidence to support these practices ${ }^{104,105}$ and the general support for BAL by many experienced groups as the preferred sampling technique. ${ }^{97,99,100,102,103}$ Although there is evidence for low-volume BAL in diagnosing infections, ${ }^{107,108}$ based on Section 1 explanations, a low-volume airway sample does not necessarily reach the alveoli and should be called BW rather than BAL. Additionally, evidence to support location or side for donor lung assessment is lacking.

8.2.2. If donor airway samples are collected for research, should the sampling be altered?. Based on the ISHLT BAL survey, most centers $(86.7 \%)$ do not collect donor bronchial samples for research; however, $6.7 \%$ do so for specific studies and $4.8 \%$ for biobanks. In the literature, descriptions of donor lung sampling methods for research vary from low-volume BAL to standard BAL techniques. ${ }^{5,103,109-117}$ In general, studies in which the organ was ultimately used for transplant used less saline for sample collection ${ }^{5,103,109-112,114-117}$ than those that used organs for pure research. ${ }^{113,118}$ In all studies that reported research methodology for sample collections of the donor airway in LTx, there was consistent agreement in wedged sampling from a single segment. ${ }^{5,103,109-117}$ Location of sampling varied between studies but most commonly was from the right middle lobe. ${ }^{5,103,109-117}$ Dwell time was uniformly not reported.

\section{Statement}

- For donor lungs destined for clinical use, this expert consensus panel recommends performing BW airway sampling according to statement 8.2.1. For donor lungs destined for research, we recommend choosing either the donor lung BW sampling strategy (statement 8.2.1) or the recipient BAL sampling strategy (statement 1.3). (C1, S2, 0I, V95\%)

8.2.3. Are there special considerations in the context of clinical ex vivo lung perfusion (EVLP)? . Evidence defining when and how to perform airway sampling in EVLP cases is limited. Most centers perform bronchial sampling before EVLP (17.1\%) rather than during $(8.6 \%)$ or after EVLP $(6.7 \%)$. Within the literature, sampling before and after EVLP has been described when used to define changes to organs on EVLP. ${ }^{110,111,115}$

\section{Statement}

- Insufficient evidence is available to define the ideal timing for airway sample collection with respect to EVLP; however, assessment of the donor should take place as part of routine donor evaluation regardless of EVLP use. We recommend BW sampling before donor organ retrieval, performed according to statement 8.2.1. During or post-EVLP bronchoscopic sample collections can be performed for study-specific uses. (C1, S5, 0I, V94\%) 
8.2.4. How should the airway samples be transported from the donor to the recipient hospital? Based on the ISHLT BAL survey, for research purposes, donor bronchial samples are usually transported on ice or at $4^{\circ} \mathrm{C}(58.3 \%)$ or room temperature $(16.7 \%)$. Given the limitations imposed by transport from a remote donor location to the transplant center, the simplest most inexpensive means for $\mathrm{BAL} / \mathrm{BW}$ sample preservation is to use the cooler for organs and to keep the sample at $4^{\circ} \mathrm{C}$. $5,97,109,114$

\section{Statement}

- Direct comparisons of methods for preservation and transport are currently not available. We recommend transport of donor airway samples on ice or at $4^{\circ} \mathrm{C}$. (C1, S4, 0I, v94\%)

\subsection{What clinical studies should be ordered on the airway samples obtained from donors?}

Based on the ISHLT BAL survey, clinical donor assessment of airway samples most commonly includes bacterial Gram stain and culture $(75.2 \%)$, fungal stain and culture $(59 \%)$, and acid-fast bacilli stain and culture (49.5\%). Practices cited in the literature agree that microbiological assessment is necessary, ${ }^{28,97,99-101,103,104,106,118}$ but unambiguous description of the specific clinical assessment is sometimes lacking. In general, most suggest that bacterial Gram stain and culture and fungal stain and culture should be performed. ${ }^{97,103,106,115}$ Most transplant centers (73.3\%) do not use viral assessment as part of their minimum clinical analysis. Only 1 manuscript $^{97}$ and $12 \%$ of transplant programs have described viral assessment as part of routine organ evaluation.

\section{Statements}

- There is broad agreement that microbiological assessment of donor lungs should be performed. (C1, S2, OI, V99\%)

- Data regarding specific clinical microbiological studies are limited, but international consensus and the available literature support bacterial Gram stain and culture as a minimum clinical assessment. (C1, S2, OI, v94\%)

- Fungal and mycobacterial stain and culture have limited support within the literature but are part of most transplant centers' practices; we recommend including them in the standard donor airway microbiological assessment. (C1, S3, OI, V96\%)

- Evidence for viral assessment of donor airway samples is limited. It is recommended that use of viral studies should be individualized to specific situations with high index of suspicion. (C1, S5, OI, V88\%)

\section{Pediatric-specific considerations}

With no established standards in children after LTx, pediatric-specific recommendations in this document are based on the ISHLT BAL survey and the expert opinion of the consensus panel.

\subsection{Indications and contraindications for $B A L$ sampling in children after LTX}

With evidence that children often have silent allograft rejection or subclinical infection, especially during the first year after $\mathrm{LTx}^{11}$ routine surveillance bronchoscopy is widely used according to published reports in pediatric LTX recipients. $^{119}$ Of the 8 pediatric LTx centers surveyed, $100 \%$ of centers reported performing surveillance bronchoscopies with BAL. In addition, diagnostic bronchoscopy is universally performed when clinical evidence suggests a deterioration in allograft health from infection, AR, AMR, or CLAD. At a few select transplant programs where infants undergo LTx, surveillance and diagnostic bronchoscopy are performed even in the youngest post-LTx patient population, ${ }^{119,120}$ so there are no age or size limitations.

Although contraindications to BAL were not included in an official document for pediatric airway endoscopy, ${ }^{121}$ common complications include bronchospasm, bleeding, hypotension, hypoxemia, and tachycardia from either the procedure or sedation. Building upon these standards, our group's consensus is that contraindications for BAL collection include conditions where a risk-benefit ratio is not favorable for pediatric LTx recipients.

\section{Statement}

- The collection of BAL fluid is indicated in pediatric LTx recipients of all ages undergoing surveillance and diagnostic bronchoscopy for assessment of the allograft for infection and/or rejection. The potential complications associated with this procedure have to be evaluated carefully and taken into consideration. (C1, S1, 0I, V96\%)

\subsection{Methods for performing BAL in children after $L T x$}

The technical standards for performing bronchoscopy and BAL in children were recently developed and published by an ad hoc committee of ATS. ${ }^{121}$ Expanding upon these standards, we identified relevant issues specific to the pediatric LTx population.

The longitudinal assessment is usually performed at predetermined times during the first year post-LTx. The ISHLT BAL survey identified that surveillance bronchoscopies with BAL were universally performed by 2 weeks and at 3 , 6 , and 12 months post-LTx, with some variability for other 
time points. Additional time points included by 6 weeks ( 4 of 8 centers) and at 9 months ( 4 of 8 centers) post-LTx, resulting in 6 surveillance bronchoscopies with BAL during the first post-LTx year in most pediatric LTx recipients. After the first post-LTx year, surveillance bronchoscopies with BAL were performed less widely with 3 of 8 centers, 2 of 8 centers, and 2 of 8 centers reporting 18 month, 24 month, and yearly procedures, respectively.

Collection of BAL was universally performed before transbronchial biopsies ( 8 of 8 centers), although most collected BAL in one allograft (5 of 8 centers). For surveillance bronchoscopy with BAL, the preferred location was the middle lobe ( 8 of 8 centers) and the lingula ( 7 of 8 centers). According to the ISHLT BAL survey, all 8 pediatric centers instill $1 \mathrm{ml} / \mathrm{kg}$ to perform a BAL. Our consensus group determined the maximum volume to be that recommended for the adult population, that is, $50 \mathrm{ml}$ for children of $50 \mathrm{~kg}$ or greater. The ISHLT BAL survey identified that most pediatric LTx experts use no dwell time before aspirating the BAL (5 of 8 centers). Previous research determined manual suctioning during bronchoscopy was associated with a higher percentage of BAL volume return and increased odds of performing technically acceptable procedures in children. ${ }^{122}$ Consistent with this research and other reports, ${ }^{119}$ the survey identified that pediatric LTx centers performing bronchoscopy with BAL in children after LTx use manual suctioning with a syringe (5 of 8 centers).

\section{Statements}

In addition to following established technical standards in performing bronchoscopy and collecting BAL in children, specific considerations are provided to address LTx-specific issues for BAL fluid collection in pediatric LTX recipients:

- Surveillance bronchoscopies should be considered for the first year post-transplant. Diagnostic bronchoscopies are recommended when clinically indicated as determined by monitoring of lung allograft health with pulmonary function testing and chest imaging. (C1, S1, 0I, 93\%)

- We recommend minimum time points for surveillance bronchoscopies with BAL to include 1, 3, 6, and 12 months post-transplant; consideration for surveillance bronchoscopies with BAL at additional time points may be needed because of the difficulty in performing surveillance pulmonary function tests in this high-risk patient population (as opposed to the adult population). (C1, S2, 0I, V86\%)

- BAL fluid collection during surveillance bronchoscopies should be done unilaterally, using preferentially manual suction, before performing transbronchial lung biopsies. (C2, S2, 0I, V91\%)
- Recommended locations of BAL fluid collection during surveillance bronchoscopies are middle lobe or lingula, unless otherwise directed by abnormal imaging or airway examination. (C2, S1, OI, V92\%)

- The recommended BAL volume of instilled sterile saline is $1 \mathrm{ml} / \mathrm{kg}$ per aliquot up to $50 \mathrm{ml}$ per aliquot for children $50 \mathrm{~kg}$ or greater (consistent with the adult BAL recommendations), using a maximum of 2 aliquots. (C1, S1, 0I, V88\%)

- Immediate aspiration of BAL, once sterile saline is instilled, is recommended (i.e., no dwell time). (C2, S2, 0I, V94\%)

\subsection{Analysis of BAL in pediatric lung transplant recipients}

Following standard laboratory protocols for handling BAL after collection, the ISHLT BAL survey identified that routine analysis of BAL in pediatric LTx recipients included total cell count with differential counts ( 8 of 8 centers), cytology ( 8 of 8 centers), and Oil-Red-O stain for aspiration ( 7 of 8 centers). A wide array of microbiologic testing is also performed for bacteria ( 8 of 8 centers), fungi ( 7 of 8 centers), mycobacteria (7 of 8 centers), and respiratory viruses (influenza, parainfluenza, adenovirus, RSV, rhinovirus, and human metapneumovirus) (7 of 8 centers). When available, PCR or other molecular techniques are preferred for PJP ( 6 of 8 centers), CMV (6 of 8 centers), and respiratory viruses ( 7 of 8 centers). Although not all pediatric LTx programs surveyed perform research using BAL from recipients ( 5 of 8 centers), the 5 centers that do collect BAL for research split the fluid for clinical and research purposes (5 of 5 centers).

\section{Statements}

In addition to following established standards for handling and transporting BAL fluid from children, specific considerations are provided to address transplant-specific issues for BAL analysis in pediatric LTx recipients:

- BAL analysis should include total cell and differential cell counts, including eosinophils, macrophages, lymphocytes, and neutrophils. (C1, S1, 0I, V88\%)

- BAL analysis should include bacterial (CF respiratory culture when appropriate), fungal, and mycobacterial staining, galactomannan, and cultures. (C1, S1, OI, V88\%)

- BAL analysis should include PCR for common respiratory viruses. PCR for CMV and PJP should be considered when clinically appropriate. (C1, S1, 0I, V88\%)

- We recommend splitting of BAL for research purposes if sufficient sample is obtained to complete clinical analysis. (C2, S1, 0I, V94\%) 


\subsection{Analysis of $B A L$ and $B W$ in pediatric lung donors}

The medical literature supports the use of bronchoscopy with BAL for assessing pediatric donor lungs. ${ }^{123}$ Donor bronchoscopy allows for inspection of the airway and clearance of secretions, and the BAL or BW microbiological data can help guide antimicrobial therapy post-LTx. Although repeated bronchoscopy may be needed in some donors to manage secretions, consideration should be given to the potential risk of causing lung injury. The ISHLT BAL survey did not identify specifics about airway sampling for pediatric donors, so the pediatric experts on the ISHLT consensus panel recommended limiting fluid instillations to smaller aliquots.

\section{Statements}

- Given the lack of evidence and practice variation, no airway sampling technique or volume appears superior for clinical pediatric donor lung assessment. Thus, transplant-specific considerations are based on expert opinion of the consensus panel: Donor airway samples usually involve low-volume instillations, which are unlikely to reach alveolar spaces, and should therefore be called BW samples instead of BAL (as explained in Section 1). Our recommended BW volume of instilled sterile saline for pediatric lung donors is $0.5 \mathrm{ml} / \mathrm{kg}$, up to a maximum of $20 \mathrm{ml}$ per aliquot (consistent with the adult donor airway sampling recommendation). If the yield is insufficient, up to 3 aliquots can be used to allow for optimal sampling, while aiming to prevent development of new opacities on radiography or transient hypoxemia, which may interfere with donor evaluation. (C2, S1, OI, V89\%)

\section{Conclusion}

Based on a comprehensive international survey of lung transplant centers and the best available evidence, the ISHLT BAL standardization workforce herein puts forth recommendations for BAL collection and processing in LTx. The literature review identified limited data to inform certain statements, emphasizing the need for further studies to better direct future revisions of this document. In spite of this limitation, the statements represent a consensus approach that can serve to standardize practice within the community. Members of the ISHLT BAL standardization workforce hope that this document will harmonize clinical and research practices for BAL collection and processing in LTx. The overarching goal is to enhance standardization and multicenter collaboration within the international lung transplant community and enable improvement and development of new BAL-based diagnostics.

\section{Members of the Workforce}

Co-leaders: Tereza Martinu, Angela Koutsokera, Shaf Keshavjee

Subgroup 1: S. Samuel Weigt, Masaaki Sato, Cecilia Chaparro, David Wilson Roe, Frank D'Ovidio, George Chaux, Greg Snell, Laurent Godinas, Mohamed Al-Aloul, Steven Hays

Subgroup 2: Jamie Todd, Michael Perch, Amy Rigby, Louis Clauden, Matthew Morrell, Puneet Garcha, Sanjeev Raman, Soma Jyothula, Michael Trotter

Subgroup 3: Erika Lease, Jeffrey Edelman, Cassie Kennedy, Chadi A Hage, Saima Aslam, Shahid Husain

Subgroup 4: Jan von der Thüsen, Andrew J. Fisher, Katharina Wassilew, Reinaldo Rampolla-Selles, Siddhartha G Kapnadak, Umesh Goswami

Subgroup 5: John Greenland, Amir Emtiazjoo, Aric Gregson, Bart Vanaudenaerde, Tji Gan

Subgroup 6: David Hwang, Brian Keller, Laura K Frye, Margaret Hannan, Harish Seethamraju, Rade Tomic, Remzi Bag, Alicia Mitchell

Subgroup 7: Stijn Verleden, Daniel Chambers, Jorge Mallea, Maria Crespo, Sangeeta Bhorade

Subgroup 8: Cantu Edward, Cypel Marcelo, Gundeep Dhillon, Jason Christie, Jessica GY Luc, Shaf Keshavjee, Keith M Wille, Olufemi Akindipe, Omar Mohamedaly, Christopher Wigfield

Subgroup 9: Don Hayes, Christian Benden, Ernestina Melicoff-Portillo, Marc Schecter, Shailendra Das

Literature search: Ani Orchanian-Cheff

Statistical analysis: George Tomlinson

\section{Disclosure statement}

The disclosures of the workforce participants are provided in Section D of the Supplementary Material.

No specific funding was available for this project. During this project, Angela Koutsokera received grants from the Swiss National Science Foundation (grants P300PB_164733 and P3P3PB_164734/1), the University Hospital of Lausanne (Fond de Perfectionnement), the Ligue Pulmonaire Suisse (grant 2018-16), and the University of Lausanne (grant Pépinière). The funding sources for each workforce member had no role in study design, data collection, data analysis, or the writing of the report.

We would like to thank the following lung transplant specialists for sharing their practices regarding bronchoalveolar lavage collection and processing and for taking the time to complete the comprehensive International Society for Heart and Lung Transplantation bronchoalveolar lavage survey which allowed the creation of this consensus document:

Adrien Tissot, Alan Betensley, Alicia De Pablo, Allison Carroll, Alim Hirji, Amir Emtiazjoo, Amparo Sole, Amy Rigby, Andrew J. Fisher, Anne Raisanen-Sokolowski, Anne Gigandon, Annie Jack, Antoine Magnan, Antoine Roux, Antonio Roman Brota, Are Martin Holm, Arun Nair, Aurelie Fabre, Bart Vanaudenaerde, Brendan O'Sallivan, Brian Keller, Burkhard Bewig, Carol Conrad, Cassie Kennedy, Catriona McLean, Chadi A. Hage, Charles Poirier, 
Christian Benden, Christiane Knoop, Christopher Wigfield, Ciara Shaver, Cindy McGrath, Claire Dromer, Claudia Sepulveda, Cynthia Gries, Daniel Chambers, Daniel F. Dilling, David Neujahr, Don Hayes, Eliska Bebrova, Eric Bernasconi, Erika Lease, Ernestina Melicoff, Esteban Wainstein, Eva Tejerina, Fenshi Chen, Fernando Torres, Francisco Santos-Luna, Frank D’Ovidio, Gary Visner, George Chaux, Gerdt Riise, Glen Westall, Greg Calligaro, Greg Snell, Hanneke Kwakkel-van Erp, Heather M. Strah, Helen Spencer, Helle Krogh Johansen, Hilary Goldberg, Hisashi Oishi, H. G. Otten, Imran Nizami, Ize Imai, Jamie Todd, Jan Havlin, Jan von der Thüsen, Jasvir Parmar, Jeffrey Edelman, Jens Gottlieb, Jerome Le Pavec, Jianyu Rao, Jiri Pozniak, Joanna Schaenman, Johanna Claustre, Johanna Kwakkel-Van Erp, John Belberio, John Greenland, JohnDavid Aubert, Jonathan Heath, Jonathan Visentin, Jorge Mallea, José Cifrian, Joshua Blatter, Joshua Diamond, Joshua Malo, Juan Braga Menendez, June Kim, Kapilkumar Patel, Karen Eldin, Katharina Wassilew, Katia Jaton, Keith M. Wille, Kim Hanson, Kirk Jones, Konstantin Mayer, Laura K. Frye, Laurel Glaser, Lennart Hansson, Leonard Jason Lobo, Macé Schuurmans, Magali Dodémont, Maher Baz, Maija Halme, Marc Schecter, Marcel Simon, Margaret Hannan, Marget Savisaar, Maria Crespo, Mark O'Carroll, Martin Goddard, Martin Zamora, Matthew Morrell, Masaaki Sato, Masayuki Chida, Massimo Bongiovanni, Matthew Morrell, Max M. Weder, Melinda Solomon, Michael Musk, Michael Perch, Miquel Ekkelenkamp, Mordechai Kramer, Nicholas Nowacki, Olivia Peuchant, Orla Morrissey, Osnat Shtraichman, Paul Williams, Patrick von Samson-Himmelstjerna, Paul Williams, Paula Revell, Paulo Manuel Pego-Fernandes, Peter Dorfmuller, Peter Jaksch, Peter Riddell, Piedad Ussetti Gil, Preeti Pancholi, Puneet Garcha, Rade Tomic, Rajat Walia, Ramsey Hachem, Reda Girgis, Reinaldo Rampolla-Selles, Richard Thompson, Robert Levy, Rogier Hoek, Romain Kessler, Sacha Mussot, Shailendra Das, S. Samuel Weigt, Samer Hakmi, Samuel Goldfarb, Sangeeta Bhorade, Sanjeev Raman, Seiichiro Sugimto, Sivagini Ganesh, Shahid Husein, Soma Jyothula, Song Yee Kim, Spencer Camargo, Steve Miller, Steven Peart, Stijn Verleden, Takahiro Nakajima, Takeshi Shiraishi, Tereza Martinu, Thomas Kaleekal, Tji Gan, Toyofumi Chen-Yoshikwa, Troy Matthias, TS Kaleekal, Yvonne Yau, and Zeling Cao.

We thank Mrs Megan Barrett for providing administrative support and for helping with the preparation and distribution of the surveys used for this project.

We also thank the expert reviewers for their input.

\section{Supplementary data}

Supplementary data associated with this article can be found in the online version at www.jhltonline.org/.

\section{Supplementary materials}

Supplementary material associated with this article can be found in the online version at https://doi.org/10.1016/j. healun.2020.07.006.

\section{References}

1. Kennedy VE, Todd JL, Palmer SM. Bronchoalveolar lavage as a tool to predict, diagnose and understand bronchiolitis obliterans syndrome. Am J Transplant 2013;13:552-61.

2. Speck NE, Schuurmans MM, Benden C, Robinson CA, Huber LC Plasma and bronchoalveolar lavage samples in acute lung allograft rejection: the potential role of cytokines as diagnostic markers. Respir Res 2017;18:151.

3. Speck NE, Schuurmans MM, Murer C, Benden C, Huber LC. Diagnostic value of plasma and bronchoalveolar lavage samples in acute lung allograft rejection: differential cytology. Respir Res 2016;17:74.

4. Greenland JR, Jewell NP, Gottschall M, et al. Bronchoalveolar lavage cell immunophenotyping facilitates diagnosis of lung allograft rejection. Am J Transplant 2014;14:831-40.

5. Cantu E, Lederer DJ, Meyer K, et al. Gene set enrichment analysis identifies key innate immune pathways in primary graft dysfunction after lung transplantation. Am J Transplant 2013;13:1898-904.

6. Haslam PL, Baughman RP. Report of ERS Task Force: guidelines for measurement of acellular components and standardization of BAL. Eur Respir J 1999;14:245-8.

7. Meyer KC, Raghu G, Baughman RP, et al. An official American Thoracic Society clinical practice guideline: the clinical utility of bronchoalveolar lavage cellular analysis in interstitial lung disease. Am J Respir Crit Care Med 2012;185:1004-14.

8. Du Rand IA, Blaikley J, Booton R, et al. British Thoracic Society guideline for diagnostic flexible bronchoscopy in adults: accredited by NICE. Thorax 2013;68(Suppl 1):i1-i44

9. Landis JR, Koch GG. The measurement of observer agreement for categorical data. Biometrics 1977;33:159-74.

10. Rouby JJ, Rossignon MD, Nicolas MH, et al. A prospective study of protected bronchoalveolar lavage in the diagnosis of nosocomial pneumonia. Anesthesiology 1989;71:679-85.

11. Benden C, Harpur-Sinclair O, Ranasinghe AS, Hartley JC, Elliott MJ, Aurora P. Surveillance bronchoscopy in children during the first year after lung transplantation: is it worth it? Thorax 2007;62:57-61.

12. Girgis RE, Reichenspurner H, Robbins RC, Reitz BA, Theodore J. The utility of annual surveillance bronchoscopy in heart-lung transplant recipients. Transplantation 1995;60:1458-61.

13. Guilinger RA, Paradis IL, Dauber JH, et al. The importance of bronchoscopy with transbronchial biopsy and bronchoalveolar lavage in the management of lung transplant recipients. Am J Respir Crit Care Med 1995;152:2037-43.

14. Inoue M, Minami M, Wada N, et al. Results of surveillance bronchoscopy after cadaveric lung transplantation: a Japanese singleinstitution study. Transplant Proc 2014;46:944-7.

15. McWilliams TJ, Williams TJ, Whitford HM, Snell GI. Surveillance bronchoscopy in lung transplant recipients: risk versus benefit J Heart Lung Transplant 2008;27:1203-9.

16. Valentine VG, Gupta MR, Weill D, et al. Single-institution study evaluating the utility of surveillance bronchoscopy after lung transplantation. J Heart Lung Transplant 2009;28:14-20.

17. Glanville AR. Bronchoscopic monitoring after lung transplantation. Semin Respir Crit Care Med 2010;31:208-21.

18. Lehto JT, Koskinen PK, Anttila VJ, et al. Bronchoscopy in the diagnosis and surveillance of respiratory infections in lung and heartlung transplant recipients. Transpl Int 2005;18:562-71.

19. Rademacher J, Suhling H, Greer M, et al. Safety and efficacy of outpatient bronchoscopy in lung transplant recipients - a single centre analysis of 3,197 procedures. Transplant Res 2014;3:11.

20. Weiss SM, Hert RC, Gianola FJ, Clark JG, Crawford SW. Complications of fiberoptic bronchoscopy in thrombocytopenic patients. Chest 1993; 104:1025-8

21. Chhajed PN, Aboyoun C, Malouf MA, et al. Management of acute hypoxemia during flexible bronchoscopy with insertion of a nasopharyngeal tube in lung transplant recipients. Chest 2002;121:1350-4.

22. Albertini RE, 2nd Harrell JH, Kurihara N, Moser KM. Arterial hypoxemia induced by fiberoptic bronchoscopy. JAMA 1974;230:1666-7.

23. Kirkpatrick MB. Lidocaine topical anesthesia for flexible bronchoscopy. Chest 1989;96:965-7. 
24. Wimberley N, Willey S, Sullivan N, Bartlett JG. Antibacterial properties of lidocaine. Chest 1979;76:37-40.

25. Aslam S, Danziger-Isakov L, Luong ML, et al. Guidance from the International Society of Heart and Lung Transplantation regarding the SARS CoV-2 pandemic. Available at: https://ishlt.org/ishlt/media/ documents/SARS-CoV-2_-Guidance-for-Cardiothoracic-Transplantand-VAD-centers.pdf.

26. Wahidi MM, Shojaee S, Lamb CR, et al. The use of bronchoscopy during the COVID-19 pandemic: CHEST/AABIP guideline and Expert Panel report [e-pub ahead of print]. Chest. doi: 10.1016/j. chest.2020.04.036, accessed April 30, 2020.

27. Limper AH, Specks U, Brutinel WM, 2nd Martin WJ, Rohrbach MS. Interlobar variation in the recovery of bronchoalveolar lavage fluid, cell populations, and angiotensin-converting enzyme in normal volunteers. J Lab Clin Med 1993;121:785-91.

28. Chhajed PN, Tamm M, Glanville AR. Role of flexible bronchoscopy in lung transplantation. Semin Respir Crit Care Med 2004;25:413-23

29. Davis GS, Giancola MS, Costanza MC, Low RB. Analyses of sequential bronchoalveolar lavage samples from healthy human volunteers. Am Rev Respir Dis 1982;126:611-6.

30. Dohn MN, Baughman RP. Effect of changing instilled volume for bronchoalveolar lavage in patients with interstitial lung disease. Am Rev Respir Dis 1985;132:390-2.

31. Merrill W, O’Hearn E, Rankin J, Naegel G, Matthay RA, Reynolds HY. Kinetic analysis of respiratory tract proteins recovered during a sequential lavage protocol. Am Rev Respir Dis 1982;126:617-20.

32. Kelly CA, Kotre CJ, Ward C, Hendrick DJ, Walters EH. Anatomical distribution of bronchoalveolar lavage fluid as assessed by digital subtraction radiography. Thorax 1987;42:624-8.

33. Levy L, Juvet SC, Boonstra K, et al. Sequential broncho-alveolar lavages reflect distinct pulmonary compartments: clinical and research implications in lung transplantation. Respir Res 2018;19:102.

34. Rennard SI, Basset G, Lecossier D, et al. Estimation of volume of epithelial lining fluid recovered by lavage using urea as marker of dilution. J Appl Physiol (1985) 1986;60:532-8.

35. Rennard SI, Ghafouri M, Thompson AB, et al. Fractional processing of sequential bronchoalveolar lavage to separate bronchial and alveolar samples. Am Rev Respir Dis 1990;141:208-17.

36. Baughman RP. Technical aspects of bronchoalveolar lavage: recommendations for a standard procedure. Semin Respir Crit Care Med 2007; 28:475-85

37. Chambers DC, Cherikh WS, Goldfarb SB, et al. The International Thoracic Organ Transplant Registry of the International Society for Heart and Lung Transplantation: thirty-fifth adult lung and heartlung transplant report-2018; focus theme: multiorgan transplantation. J Heart Lung Transplant 2018;37:1169-83.

38. Belperio J, Palmer SM, Weigt SS. Host-pathogen interactions and chronic lung allograft dysfunction. Ann Am Thorac Soc 2017;14 (Suppl 3):S242-6.

39. Campos S, Caramori M, Teixeira R, et al. Bacterial and fungal pneumonias after lung transplantation. Transplant Proc 2008;40:822-4.

40. Gottlieb J, Schulz TF, Welte T, et al. Community-acquired respiratory viral infections in lung transplant recipients: a single season cohort study. Transplantation 2009;87:1530-7.

41. Kovats Z, Sütto Z, Muraközy G, et al. Airway pathogens during the first year after lung transplantation: a single-center experience. Transplant Proc 2011;43:1290-1.

42. Miller JM, Binnicker MJ, Campbell S, et al. A guide to utilization of the microbiology laboratory for diagnosis of infectious diseases: 2018 update by the Infectious Diseases Society of America and the American Society for Microbiology. Clin Infect Dis 2018;67:e1-e94.

43. Al-Za'abi AM, MacDonald S, Geddie W, Boerner SL. Cytologic examination of bronchoalveolar lavage fluid from immunosuppressed patients. Diagn Cytopathol 2007;35:710-4.

44. Wanner TJ, Gerhardt SG, Diette GB, Rosenthal DL, Orens JB. The utility of cytopathology testing in lung transplant recipients. J Heart Lung Transplant 2005;24:870-4.
45. Walts AE, Marchevsky AM, Morgan M. Pulmonary cytology in lung transplant recipients: recent trends in laboratory utilization. Diagn Cytopathol 1991;7:353-8

46. Ohori NP. Epithelial cell atypia in bronchoalveolar lavage specimens from lung transplant recipients. Am J Clin Pathol 1999;112:204-10

47. Hopkins PM, Kermeen F, Duhig E, et al. Oil Red O stain of alveolar macrophages is an effective screening test for gastroesophageal reflux disease in lung transplant recipients. J Heart Lung Transplant 2010;29:859-64.

48. Selvaggi SM. Bronchoalveolar lavage in lung transplant patients. Acta Cytol 1992;36:674-9.

49. Slebos DJ, Postma DS, Koëter GH, Van Der Bij W, Boezen M, Kauffman HF. Bronchoalveolar lavage fluid characteristics in acute and chronic lung transplant rejection. J Heart Lung Transplant 2004:23:532-40

50. Tikkanen J, Lemström K, Halme M, Pakkala S, Taskinen E, Koskinen P. Cytological monitoring of peripheral blood, bronchoalveolar lavage fluid, and transbronchial biopsy specimens during acute rejection and cytomegalovirus infection in lung and heart-lung allograft recipients. Clin Transplant 2001;15:77-88.

51. Neurohr C, Huppmann P, Samweber B, et al. Prognostic value of bronchoalveolar lavage neutrophilia in stable lung transplant recipients. J Heart Lung Transplant 2009;28:468-74.

52. Reynaud-Gaubert M, Marin V, Thirion X, et al. Upregulation of chemokines in bronchoalveolar lavage fluid as a predictive marker of post-transplant airway obliteration. J Heart Lung Transplant 2002;21:721-30.

53. Reynaud-Gaubert M, Thomas P, Badier M, Cau P, Giudicelli R, Fuentes P. Early detection of airway involvement in obliterative bronchiolitis after lung transplantation. Functional and bronchoalveolar lavage cell findings. Am J Respir Crit Care Med 2000;161:1924-9.

54. Verleden SE, Ruttens D, Vandermeulen E, et al. Predictors of survival in restrictive chronic lung allograft dysfunction after lung transplantation. J Heart Lung Transplant 2016;35:1078-84.

55. Verleden SE, Ruttens D, Vandermeulen E, et al. Elevated bronchoalveolar lavage eosinophilia correlates with poor outcome after lung transplantation. Transplantation 2014;97:83-9.

56. Anderson RL, Hiemstra PS, Ward C, et al. Antimicrobial peptides in lung transplant recipients with bronchiolitis obliterans syndrome. Eur Respir J 2008;32:670-7.

57. Kotsimbos AT, Sinickas V, Glare EM, et al. Quantitative detection of human cytomegalovirus DNA in lung transplant recipients. Am J Respir Crit Care Med 1997;156:1241-6.

58. Whitehead BF, Stoehr C, Wu CJ, et al. Cytokine gene expression in human lung transplant recipients. Transplantation 1993;56:956-61.

59. Whitehead BF, Stoehr C, Finkle C, et al. Analysis of bronchoalveolar lavage from human lung transplant recipients by flow cytometry. Respir Med 1995;89:27-34.

60. Whitehead B, Scott JP, Helms P, et al. Technique and use of transbronchial biopsy in children and adolescents. Pediatr Pulmonol 1992;12:240-6.

61. Wood KL, Nunley DR, Moffatt-Bruce S, et al. The role of heat shock protein 27 in bronchiolitis obliterans syndrome after lung transplantation. J Heart Lung Transplant 2010;29:786-91.

62. Bollmann BA, Seeliger B, Drick N, Welte T, Gottlieb JT, Greer M. Cellular analysis in bronchoalveolar lavage: inherent limitations of current standard procedure. Eur Respir J 2017;49:1601844

63. Mohammed A, Ulukpo O, Lawrence EC, et al. Cumulative exposure to $\mathrm{CD} 8+$ granzyme Bhi $\mathrm{T}$ cells is associated with reduced lung function early after lung transplantation. Transplant Proc 2011;43: 3892-8.

64. Neujahr DC, Cardona AC, Ulukpo O, et al. Dynamics of human regulatory $\mathrm{T}$ cells in lung lavages of lung transplant recipients. Transplantation 2009;88:521-7.

65. Neujahr DC, Mohammed A, Ulukpo O, et al. Surgical correction of gastroesophageal reflux in lung transplant patients is associated with decreased effector CD8 cells in lung lavages: a case series. Chest 2010;138:937-43. 
66. Stéphan F, Fajac A, Grenet D, et al. Predictive value of cytomegalovirus DNA detection by polymerase chain reaction in blood and bronchoalveolar lavage in lung transplant patients. Transplantation 1997;63:1430-5.

67. Steinhoff G, Behrend M, Wagner TO, Höper MH, Haverich A. Early diagnosis and effective treatment of pulmonary CMV infection after lung transplantation. J Heart Lung Transplant 1991;10:9-14.

68. Kelly FL, Kennedy VE, Jain R, et al. Epithelial Clara cell injury occurs in bronchiolitis obliterans syndrome after human lung transplantation. Am J Transplant 2012;12:3076-84

69. Walker N, Badri L, Wettlaufer S, et al. Resident tissue-specific mesenchymal progenitor cells contribute to fibrogenesis in human lung allografts. Am J Pathol 2011;178:2461-9.

70. Stovold R, Forrest IA, Corris PA, et al. Pepsin, a biomarker of gastric aspiration in lung allografts: a putative association with rejection. Am J Respir Crit Care Med 2007;175:1298-303.

71. Klech H, Pohl W. Technical recommendations and guidelines for bronchoalveolar lavage (BAL). Report of the European Society of Pneumology Task Group. Eur Respir J 1989;2:561-85.

72. Costa C, Curtoni A, Bergallo M, et al. Quantitative detection of HHV-6 and HHV-7 in transbronchial biopsies from lung transplant recipients. New Microbiol 2011;34:275-80.

73. Vanaudenaerde BM, Wuyts WA, Geudens N, et al. Broncho-alveolar lavage fluid recovery correlates with airway neutrophilia in lung transplant patients. Respir Med 2008;102:339-47.

74. Darnell ME, Subbarao K, Feinstone SM, Taylor DR. Inactivation of the coronavirus that induces severe acute respiratory syndrome, SARS-CoV. J Virol Methods 2004;121:85-91.

75. Salahuddin S, Thomson E, Méziane O, et al. Processing of bronchoalveolar lavage fluid and matched blood for alveolar macrophage and CD4+ T-cell immunophenotyping and HIV reservoir assessment. J Vis Exp 2019(148).

76. Weigt SS, Wang X, Palchevskiy V, et al. Gene expression profiling of bronchoalveolar lavage cells preceding a clinical diagnosis of chronic lung allograft dysfunction. Ann Am Thorac Soc 2017;14 (Suppl 3):S252.

77. Gunasekaran M, Xu Z, Nayak DK, et al. Donor-derived exosomes with lung self-antigens in human lung allograft rejection. Am J Transplant 2017;17:474-84.

78. Weigt SS, Derhovanessian A, Liao E, et al. CXCR3 chemokine ligands during respiratory viral infections predict lung allograft dysfunction. Am J Transplant 2012;12:477-84.

79. Mitchell AB, Glanville AR. The human respiratory microbiome: implications and impact. Semin Respir Crit Care Med 2018;39:199212.

80. Mouraux S, Bernasconi E, Pattaroni C, et al. Airway microbiota signals anabolic and catabolic remodeling in the transplanted lung. J Allergy Clin Immunol 2018;141:718-729.e7

81. Schott C, Weigt SS, Turturice BA, et al. Bronchiolitis obliterans syndrome susceptibility and the pulmonary microbiome. J Heart Lung Transplant 2018;37:1131-40

82. Sharma NS, Wille KM, Athira S, et al. Distal airway microbiome is associated with immunoregulatory myeloid cell responses in lung transplant recipients. J Heart Lung Transplant. 2018;37:206-16.

83. Beaume M, Köhler T, Greub G, et al. Rapid adaptation drives invasion of airway donor microbiota by Pseudomonas after lung transplantation. Sci Rep 2017;7:40309.

84. Syed SA, Whelan FJ, Waddell B, Rabin HR, Parkins MD, Surette MG. Reemergence of lower-airway microbiota in lung transplant patients with cystic fibrosis. Ann Am Thorac Soc 2016;13:2132-42.

85. Beaume M, Lazarevic V, Köhler T, et al. Microbial communities of conducting and respiratory zones of lung-transplanted patients. Front Microbiol 2016;7:1749.

86. Bernasconi E, Pattaroni C, Koutsokera A, et al. Airway microbiota determines innate cell inflammatory or tissue remodeling profiles in lung transplantation. Am J Respir Crit Care Med 2016;194:1252-63.

87. Shankar J, Nguyen MH, Crespo MM, et al. Looking beyond respiratory cultures: microbiome-cytokine signatures of bacterial pneumonia and tracheobronchitis in lung transplant recipients. Am J Transplant 2016;16:1766-78

88. Dickson RP, Erb-Downward JR, Prescott HC, et al. Cell-associated bacteria in the human lung microbiome. Microbiome 2014;2:28.

89. Dickson RP, Erb-Downward JR, Freeman CM, et al. Changes in the lung microbiome following lung transplantation include the emergence of two distinct Pseudomonas species with distinct clinical associations. PLoS One 2014;9:e97214.

90. Becker J, Poroyko V, Bhorade S. The lung microbiome after lung transplantation. Expert Rev Respir Med 2014;8:221-31.

91. Bittinger K, Charlson ES, Loy E, et al. Improved characterization of medically relevant fungi in the human respiratory tract using nextgeneration sequencing. Genome Biol 2014;15:487.

92. Borewicz K, Pragman AA, Kim HB, Hertz M, Wendt C, Isaacson RE. Longitudinal analysis of the lung microbiome in lung transplantation. FEMS Microbiol Lett 2013;339:57-65.

93. Willner DL, Hugenholtz P, Yerkovich ST, et al. Reestablishment of recipient-associated microbiota in the lung allograft is linked to reduced risk of bronchiolitis obliterans syndrome. Am J Respir Crit Care Med 2013;187:640-7.

94. Charlson ES, Diamond JM, Bittinger K, et al. Lung-enriched organisms and aberrant bacterial and fungal respiratory microbiota after lung transplant. Am J Respir Crit Care Med 2012;186:536-45.

95. Segal LN, Alekseyenko AV, Clemente JC, et al. Enrichment of lung microbiome with supraglottic taxa is associated with increased pulmonary inflammation. Microbiome 2013;1:19.

96. Dickson RP, Erb-Downward JR, Freeman CM, et al. Spatial variation in the healthy human lung microbiome and the adapted island model of lung biogeography. Ann Am Thorac Soc 2015;12:821-30.

97. Fisher AJ, Donnelly SC, Pritchard G, Dark JH, Corris PA. Objective assessment of criteria for selection of donor lungs suitable for transplantation. Thorax 2004;59:434-7.

98. Riou B, Guesde R, Jacquens Y, Duranteau R, Viars P. Fiberoptic bronchoscopy in brain-dead organ donors. Am J Respir Crit Care Med 1994;150:558-60.

99. Callegari G, Fracchia C. Lung transplantation and endoscopic issues. Monaldi Arch Chest Dis 2011;75:60-3.

100. de Perrot M, Snell GI, Babcock WD, et al. Strategies to optimize the use of currently available lung donors. J Heart Lung Transplant 2004;23:1127-34

101. Gabbay E, Williams TJ, Griffiths AP, et al. Maximizing the utilization of donor organs offered for lung transplantation. Am J Respir Crit Care Med 1999;160:265-71.

102. Trulock EP. Flexible bronchoscopy in lung transplantation. Clin Chest Med 1999;20:77-87.

103. Avlonitis VS, Krause A, Luzzi L, et al. Bacterial colonization of the donor lower airways is a predictor of poor outcome in lung transplantation. Eur J Cardiothorac Surg 2003;24:601-7.

104. Low DE, Kaiser LR, Haydock DA, Trulock E, Cooper JD. The donor lung: infectious and pathologic factors affecting outcome in lung transplantation. J Thorac Cardiovasc Surg 1993;106:614-21.

105. Weill D, Dey GC, Hicks RA, et al. A positive donor Gram stain does not predict outcome following lung transplantation. J Heart Lung Transplant 2002;21:555-8.

106. Zenati M, Dowling RD, Armitage JM, et al. Organ procurement for pulmonary transplantation. Ann Thorac Surg 1989;48:882-6.

107. Lacroix G, Prunet B, Bordes J, et al. Evaluation of early mini-bronchoalveolar lavage in the diagnosis of health care-associated pneumonia: a prospective study. Crit Care 2013;17:R24

108. Torrington KG, Finelli MR. Small volume bronchoalveolar lavage used in diagnosing Pneumocystis carinii pneumonia in HIV-infected patients. Chest 1995;107:1013-7

109. Abbas AA, Diamond JM, Chehoud C, et al. The perioperative lung transplant virome: torque Teno viruses are elevated in donor lungs and show divergent dynamics in primary graft dysfunction. Am J Transplant 2017;17:1313-24.

110. Andreasson A, Karamanou DM, Perry JD, et al. The effect of ex vivo lung perfusion on microbial load in human donor lungs. J Heart Lung Transplant 2014;33:910-6. 
111. Andreasson AS, Karamanou DM, Gillespie CS, et al. Profiling inflammation and tissue injury markers in perfusate and bronchoalveolar lavage fluid during human ex vivo lung perfusion. Eur J Cardiothorac Surg 2017;51:577-86.

112. Fisher AJ, Donnelly SC, Hirani N, et al. Elevated levels of interleukin-8 in donor lungs is associated with early graft failure after lung transplantation. Am J Respir Crit Care Med 2001;163:259-65.

113. Nakajima D, Cypel M, Bonato R, et al. Ex vivo perfusion treatment of infection in human donor lungs. Am J Transplant 2016;16:1229-37.

114. Pelaez A, Force SD, Gal AA, et al. Receptor for advanced glycation end products in donor lungs is associated with primary graft dysfunction after lung transplantation. Am J Transplant 2010; 10:900-7.

115. Shafaghi S, Dezfuli AA, Makki SS, et al. Microbial pattern of bronchoalveolar lavage in brain dead donors. Transplant Proc 2011;43: 422-3.

116. Strüber M, Fischer S, Niedermeyer J, et al. Effects of exogenous surfactant instillation in clinical lung transplantation: a prospective, randomized trial. J Thorac Cardiovasc Surg 2007;133:1620-5.

117. Xu Z, Sharma M, Gelman A, Hachem R, Mohanakumar T. Significant role for microRNA-21 affecting toll-like receptor pathway in primary graft dysfunction after human lung transplantation. J Heart Lung Transplant 2017;36:331-9.

118. Shafaghi S, Mortaz E, Abbasi Dezfuli A, et al. Normothermic ex vivo lung perfusion in brain-dead donors reduces inflammatory cytokines and toll-like receptor expression. Iran J Allergy Asthma Immunol 2016;15:340-54

119. Wong JY, Westall GP, Snell GI. Bronchoscopic procedures and lung biopsies in pediatric lung transplant recipients. Pediatr Pulmonol 2015;50:1406-19.

120. Hayes D Jr, Naguib A, Kirkby S, et al. Comprehensive evaluation of lung allograft function in infants after lung and heart-lung transplantation. J Heart Lung Transplant 2014;33:507-13.

121. Faro A, Wood RE, Schechter MS, et al. Official American Thoracic Society technical standards: flexible airway endoscopy in children. Am J Respir Crit Care Med 2015;191:1066-80.

122. Rosas-Salazar C, Walczak SA, Winger DG, Kurland G, Spahr JE. Comparison of two aspiration techniques of bronchoalveolar lavage in children. Pediatr Pulmonol 2014;49:978-84.

123. Mallory GB Jr, Schecter MG, Elidemir O. Management of the pediatric organ donor to optimize lung donation. Pediatr Pulmonol 2009;44:536-46. 